ARTICLE

\title{
Ultrafast photochemistry produces superbright short-wave infrared dots for low-dose in vivo imaging
}

\author{
Harrisson D. A. Santos 1,2,12, Irene Zabala Gutiérrez (D) 3,12, Yingli Shen ${ }^{1,12}$, José Lifante ${ }^{4,5,12}$, \\ Erving Ximendes (1) 1,4, Marco Laurenti (1) 3,4, Diego Méndez-González ${ }^{3}$, Sonia Melle (iD ${ }^{6}$, \\ Oscar G. Calderón (10 6 , Enrique López Cabarcos ${ }^{3}$, Nuria Fernández (10 4,5, Irene Chaves-Coira7, \\ Daniel Lucena-Agell (10 ${ }^{10}$, Luis Monge ${ }^{4,5}$, Mark D. Mackenzie ${ }^{8}$, José Marqués-Hueso9 ${ }^{9}$, Callum M. S. Jones (i) ${ }^{9}$, \\ Carlos Jacinto ${ }^{2}$, Blanca del Rosal ${ }^{11}$, Ajoy K. $\mathrm{Kar}^{8}$, Jorge Rubio-Retama (i) ${ }^{3,4 凶}$ \& Daniel Jaque (i) ${ }^{1,4 凶}$
}

Optical probes operating in the second near-infrared window (NIR-II, 1,000-1,700 nm), where tissues are highly transparent, have expanded the applicability of fluorescence in the biomedical field. NIR-II fluorescence enables deep-tissue imaging with micrometric resolution in animal models, but is limited by the low brightness of NIR-II probes, which prevents imaging at low excitation intensities and fluorophore concentrations. Here, we present a new generation of probes ( $\mathrm{Ag}_{2} \mathrm{~S}$ superdots) derived from chemically synthesized $\mathrm{Ag}_{2} \mathrm{~S}$ dots, on which a protective shell is grown by femtosecond laser irradiation. This shell reduces the structural defects, causing an 80-fold enhancement of the quantum yield. PEGylated $\mathrm{Ag}_{2} \mathrm{~S}$ superdots enable deep-tissue in vivo imaging at low excitation intensities $\left(<10 \mathrm{~mW} \mathrm{~cm}^{-2}\right)$ and doses $\left(<0.5 \mathrm{mg} \mathrm{kg}^{-1}\right)$, emerging as unrivaled contrast agents for NIR-II preclinical bioimaging. These results establish an approach for developing superbright NIR-II contrast agents based on the synergy between chemical synthesis and ultrafast laser processing.

\footnotetext{
${ }^{1}$ Fluorescence Imaging Group, Departamento de Física de Materiales, Facultad de Ciencias, Universidad Autónoma de Madrid, Madrid 28049, Spain. ${ }^{2}$ Group of Nano-Photonics and Imaging, Instituto de Física, Universidade Federal de Alagoas, Maceió-AL 57072-900, Brazil. ${ }^{3}$ Departamento de Química en Ciencias Farmacéuticas, Universidad Complutense de Madrid, Madrid 28040, Spain. ${ }^{4}$ Nanobiology Group, Instituto Ramón y Cajal de Investigación Sanitaria, Hospital Ramón y Cajal, 28034 Madrid, Spain. ${ }^{5}$ Fluorescence Imaging Group, Departamento de Fisiología, Facultad de Medicina, Universidad Autónoma de Madrid, Madrid 28029, Spain. ${ }^{6}$ Department of Optics, Complutense University of Madrid, 28037 Madrid, Spain. ${ }^{7}$ Departament of Anatomy, Histology and Neuroscience, Facultad de Medicina, Universidad Autónoma de Madrid, Madrid 28029, Spain. ${ }^{8}$ Institute of Photonics and Quantum Sciences (IPaQS), School of Engineering and Physical Sciences, Heriot-Watt University, Edinburgh EH14 4AS, UK. ${ }^{9}$ Institute of Sensors, Signals and Systems (ISSS), School of Engineering \& Physical Sciences (EPS), Heriot-Watt University, Edinburgh EH14 4AS, UK. ${ }^{10}$ Chemical and Physical Biology, Centro de Investigaciones Biologicas, Consejo Superior de Investigaciones Cientificas CIB-CSIC, Madrid 28040, Spain. ${ }^{11}$ Centre for Micro-Photonics, Faculty of Science, Engineering and Technology, Swinburne University of Technology, Mail H74 PO Box 218, Hawthorn, VIC 3122, Australia. ${ }^{12}$ These authors contributed equally: Harrisson

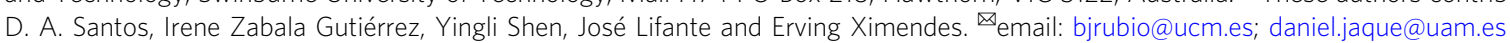


S emiconductor nanocrystals emerged almost two decades ago as novel fluorescent contrast agents capable of overcoming the intrinsic limitations of traditional fluorescent biomarkers (dyes and fluorescent proteins) ${ }^{1,2}$. The demonstration of the ability of these nanocrystals to generate high-contrast images at the cellular level was a major milestone in biophotonics and promoted further research into these materials. Optimizing their chemical stability, biocompatibility, and brightness was the major focus of the subsequent investigation ${ }^{3,4}$. That resulted in the development of core/shell architectures, which constituted the second generation of semiconductor nanocrystals ${ }^{5,6}$. These produced unparalleled results in cell imaging ${ }^{7}$, but failed to enable deep-tissue in vivo imaging as biological tissues are efficient absorbers and scatterers of visible light. This limitation was overcome by the development of a third generation of semiconductor nanocrystals, emitting in the second near-infrared window (NIR-II, $1000-1700 \mathrm{~nm})^{8}$. In this spectral range, the absorption and scattering coefficients of biological tissues are reduced to a minimum, allowing high-contrast, high-resolution in vivo imaging at large $(>1 \mathrm{~cm})$ tissue depths ${ }^{9}$. NIR-II fluorescent materials have changed the game in preclinical imaging ${ }^{10,11}$, enabling high-resolution anatomical imaging ${ }^{12,13}$, tumor detection $^{14,15}$, biosensing ${ }^{16}$, brain vasculature mapping ${ }^{17,18}$, image-guided genome editing ${ }^{19}$ and surgery ${ }^{20,21}$, and dynamic tracking of metabolic processes ${ }^{22}$. NIR-II-emitting $\mathrm{Ag}_{2} \mathrm{~S}$ nanocrystals do not contain highly toxic heavy metal ions, unlike other nanoprobes operating in this spectral range, minimizing biocompatibility concerns and making them one of the most promising systems among all currently reported NIR-II fluorophores $^{23,24}$. Their properties have been exploited for multiple applications, including subcutaneous and transcranial thermometry ${ }^{25}$, tumor theranostics via photoacoustic imaging and photothermal therapy ${ }^{26}$, early tumor diagnosis ${ }^{27}$ and dynamic imaging of the heart ${ }^{28}$ and the cardiovascular system ${ }^{29-31}$.

To enhance the potential of $\mathrm{Ag}_{2} \mathrm{~S}$ dots as NIR-II contrast agents, their properties need substantial improving to enable deep-tissue imaging at irradiation intensities well below the established safety threshold. Currently available $\mathrm{Ag}_{2} \mathrm{~S}$ dots are limited by their low fluorescence brightness, which is a result of their low quantum yield $(\mathrm{QY}<1 \%)$ and short fluorescence lifetime $(<100 \mathrm{~ns})^{32}$. These features have been attributed to the presence of surface and structural defects and to the dot-solvent interactions that favor electronic deexcitation via non-radiative pathways ${ }^{33}$. Furthermore, the high redox potential of silver ions and the high temperatures required for $\mathrm{Ag}_{2} \mathrm{~S}$ dots synthesis lead to the simultaneous formation of metallic silver nanoparticles (NPs) that could also reduce the overall brightness due to plasmon coupling events ${ }^{34}$. Currently available chemical synthesis routes have failed to avoid these non-radiative channels in $\mathrm{Ag}_{2} \mathrm{~S}$ dots. Therefore, developing alternative approaches is essential to overcome this barrier.

In this work, we present a novel methodology that permits an 80 -fold increment in the QY of $\mathrm{Ag}_{2} \mathrm{~S}$ dots. The process is based on the irradiation of chloroform-dispersed $\mathrm{Ag}_{2} \mathrm{~S}$ dots with femtosecond laser pulses, which leads to the formation of a protective $\mathrm{AgCl}$ shell. This reduces the surface traps while minimizing dotto-medium energy transfer via non-radiative events. The brightness of the NPs generated in this process is superior to that of all other currently available NIR-II contrast agents. Their improved performance enables in vivo whole body imaging, blood vessel visualization, and biodistribution tracking at ultra-low excitation intensities.

\section{Results}

Preparation and characterization of $\mathrm{Ag}_{2} \mathrm{~S}$ superdots. We fabricated $\mathrm{Ag}_{2} \mathrm{~S}$ superdots by irradiating a dispersion of chemically synthesized $\mathrm{Ag} / \mathrm{Ag}_{2} \mathrm{~S}$ heterodimers (hereafter $\mathrm{Ag}_{2} \mathrm{~S}$ dots) in $\mathrm{CHCl}_{3}$ with femtosecond laser pulses. The chemical synthesis route we used (described in Methods) yields silver NPs as a side product (see Supplementary Fig. 1). The presence of these NPs is due to the high redox potential of silver ions at the reaction temperature ${ }^{35}$. To eliminate them, a $1 \mathrm{mg} \mathrm{mL}^{-1} \mathrm{CHCl}_{3}$ dispersion of $\mathrm{Ag}_{2} \mathrm{~S}$ dots was irradiated with a Ti:Sapphire femtosecond laser operating at $808 \mathrm{~nm}$ (see details in Methods). Laser pulses trigger the transformation of $\mathrm{Ag}$ NPs into $\mathrm{AgCl}$ particles, which are colloidally unstable and easy to remove (see Supplementary Fig. 2).

Fig. 1 shows the morphological and compositional analysis of the $\mathrm{Ag}_{2} \mathrm{~S}$ dots before and after ultrafast laser irradiation. Fig. 1a depicts a high-angle annular dark-field scanning transmission electron microscopy (HAADF-STEM) image of the assynthesized $\mathrm{Ag}_{2} \mathrm{~S}$ dots. They present an elliptical morphology with an average size of $9.5 \pm 1.0 \mathrm{~nm}$ (see Supplementary Fig. 3). The NPs present an eccentrically located, electron-dense core embedded in a matrix of lower electron density. The highresolution STEM of a typical particle reveals a crystalline structure (Fig. 1b), with lattice fringes $\mathrm{d}_{-104}=2.37 \AA$ and, in the electron-dense core, $\mathrm{d}_{111}=2.30 \AA$. These well match monoclinic $\mathrm{Ag}_{2} \mathrm{~S}$ (JCPDS No. 14-0072) and cubic Ag (JCPDS 04-0783), respectively. The less electron-dense area in Fig. $1 \mathrm{~b}$ is postulated to be $\mathrm{Ag}_{2} \mathrm{~S}$, as $\mathrm{Ag}_{2} \mathrm{~S}$ has a lower density $\left(7.2 \mathrm{~g} \mathrm{~cm}^{-3}\right)$ than $\mathrm{Ag}$ $\left(10.505 \mathrm{~g} \mathrm{~cm}^{-3}\right)$. Energy-dispersive $\mathrm{X}$-ray spectroscopy (EDS) analysis, shown in Fig. 1c-e, supports this conclusion. While the $\mathrm{NP}$ core is rich in $\mathrm{Ag}, \mathrm{S}$ is mainly located in the outer part of the NPs. This anisotropic distribution of elements is depicted in the net X-ray intensity profiles shown in Fig. $1 \mathrm{~g}$ as obtained from the magnified STEM micrograph shown in Fig. 1f. In Fig. 1g, we can observe that the maximum of the X-ray intensity assigned Ag and $\mathrm{S}$ atoms arises, respectively, from the electron-dense core and the outer area. The elemental analysis of a $\mathrm{Ag}_{2} \mathrm{~S}$ dot shows a $\mathrm{Ag}: \mathrm{S}$ ratio of 74:26. All these results support the presence of a metallic $\mathrm{Ag}$ core within the $\mathrm{Ag}_{2} \mathrm{~S}$ matrix.

After ultrafast illumination with $50 \mathrm{fs}$ pulses for $90 \mathrm{~min}(9 \mathrm{~W}$ $\mathrm{cm}^{-2}$ ), the average diameter of the NPs increased from $9.5 \pm 1.0$ to $12.3 \pm 1.0 \mathrm{~nm}$, while the size of the metallic Ag core remained constant at around $5.1 \mathrm{~nm}$ (see Fig. 1h and Supplementary Fig. 3). High-resolution STEM of a representative $\mathrm{Ag}_{2} \mathrm{~S}$ dot after ultrafast laser irradiation (Fig. 1i) reveals, again, two well-differentiated crystalline regions. An electron-dense core, with lattice fringe of $\mathrm{d}_{111}=2.30 \AA$ that agrees with metallic $\mathrm{Ag}$ is embedded in a matrix with a lattice fringe of $\mathrm{d}_{110}=2.50 \AA$, corresponding to $\mathrm{Ag}_{2} \mathrm{~S}$. The presence of a 1-nm-thick shell around the NPs can also be observed in this figure. Based on the analysis of the corresponding crystal lattices, the conjunction interfaces between the metallic and the semiconductor part consist of the (110) plane of $\mathrm{Ag}_{2} \mathrm{~S}$ and (111) of $\mathrm{Ag}$, with a lattice mismatch of $8 \%$. EDS images (Fig. $1 \mathrm{j}-\mathrm{m}$ ) revealed that the resulting NPs are composed of $\mathrm{Ag}$ and $\mathrm{S}$ with an identical distribution as that observed in the as-synthesized NPs. Further, EDS analysis indicated the presence of $\mathrm{Cl}$ atoms distributed throughout the laser-irradiated $\mathrm{Ag}_{2} \mathrm{~S}$ dots (Fig. 11). This stems from the formation of a $\mathrm{AgCl}$ shell. The presence of this shell is also evidenced in the net X-ray intensity profiles shown in Fig. 1o, obtained from the magnified STEM micrograph shown in Fig. $1 n$. The elemental analysis of a lasertreated $\mathrm{Ag}_{2} \mathrm{~S}$ dot shows a $\mathrm{Ag}: \mathrm{S}: \mathrm{Cl}$ ratio of 69:24:7. Again, the excess of $\mathrm{Ag}$ would indicate the conservation of the silver-rich core characteristic of the $\mathrm{Ag}_{2} \mathrm{~S}$ dots. Further chemical and structural characterization of the samples, including X-ray diffraction, X-ray absorption near edge structure (XANES), EDS, and HAADF-STEM data of both $\mathrm{Ag}_{2} \mathrm{~S}$ dots and superdots is provided in Supplementary Figs. 4 and 5. These additional data support the conclusions extracted from Fig. 1 that the 
a

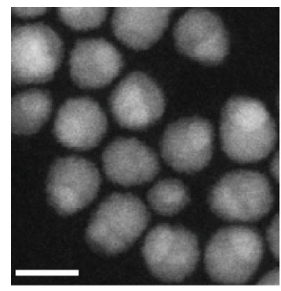

C

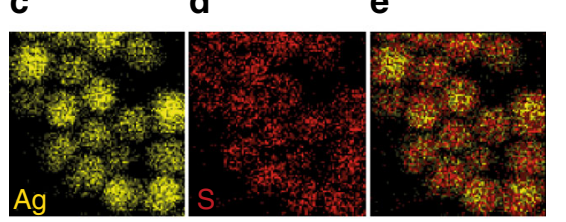

f
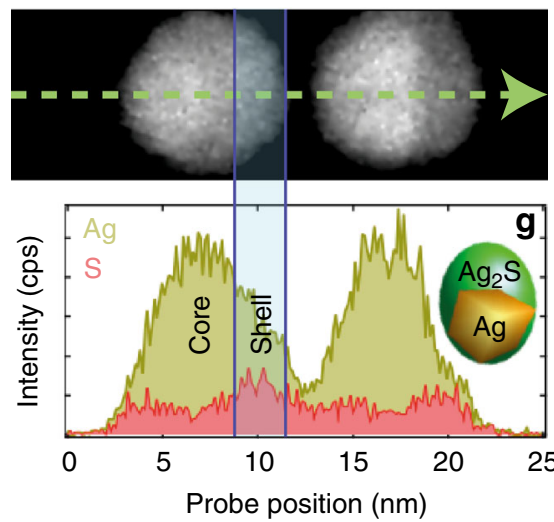

b

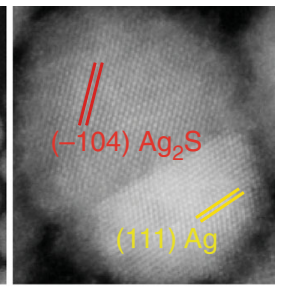

e

n
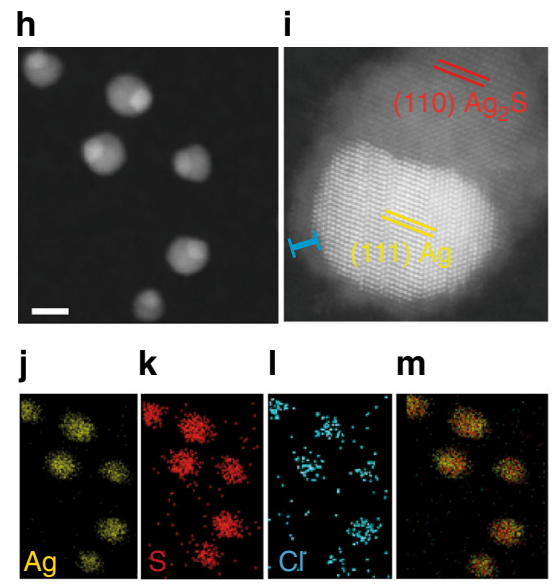

n

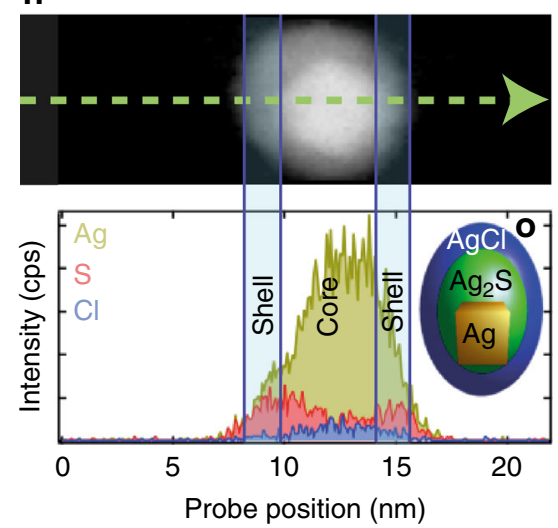

Fig. 1 Tuning structural and chemical properties through ultrafast laser irradiation. a HAADF-STEM micrograph of the as-synthesized $\mathrm{Ag}_{2} \mathrm{~S}$ dots. Scale

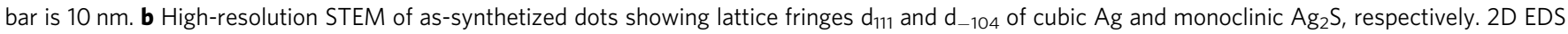
mapping of the spatial distribution of $\mathrm{Ag}(\mathbf{c}), \mathrm{S}(\mathbf{d})$, and $\mathrm{Ag}+\mathrm{S}(\mathbf{e})$. $\mathbf{f}$ Magnified STEM micrograph of two $\mathrm{Ag}_{2} \mathrm{~S}$ dots. $\mathbf{g}$ Net X-ray intensity profiles extracted from the green arrow marked in $\mathbf{f}$. Note how the $\mathrm{Ag} / \mathrm{S}$ ratio increases at the edges of the dots, which coincides with the electro-dense area. The inset shows a model $\mathrm{Ag}_{2} \mathrm{~S}$ dot. h HAADF-STEM micrograph of $\mathrm{Ag}_{2} \mathrm{~S}$ dots after ultrafast laser irradiation with 50 fs laser pulses for 90 min at a power density of $9 \mathrm{~W} \mathrm{~cm}^{-2}$. Scale bar is $10 \mathrm{~nm}$. i High-resolution STEM of an ultrafast laser-irradiated $\mathrm{Ag}_{2} \mathrm{~S}$ dot showing lattice fringes $d_{111}$ and $d_{110}$ of cubic $A g$ and monoclinic $\mathrm{Ag}_{2} \mathrm{~S}$, respectively. Note the presence of a shell marked with a blue bracket around the nanoparticle. 2D EDS mapping of the spatial distribution of $\mathrm{Ag}(\mathbf{j}), \mathrm{S}(\mathbf{k}), \mathrm{Cl}(\mathbf{I})$, and $\mathrm{Ag}+\mathrm{S}+\mathrm{Cl}(\mathbf{m})$ of an ultrafast laser-irradiated $\mathrm{Ag}_{2} \mathrm{~S}$ dot. $\mathbf{n}$ Magnified $\mathrm{STEM}$ micrograph of an ultrafast laser-irradiated $\mathrm{Ag}{ }_{2} \mathrm{~S}$ dot. - Net X-ray intensity profiles extracted from the green arrow marked in image (n), where we can observe the presence of $\mathrm{Ag}$, $\mathrm{S}$, and $\mathrm{Cl}$. The inset in o shows a schematic representation of a single $\mathrm{Ag}_{2} \mathrm{~S}$ dot after ultrafast laser irradiation.

as-synthesized dots are composed of two phases, a monoclinic $\mathrm{Ag}_{2} \mathrm{~S}$ phase and a cubic Ag phase. When these NPs are irradiated with an ultrafast laser, their chemical composition and structure change. The resulting NPs exhibit a new $\mathrm{AgCl}$ phase forming a thin outer shell, as observed in Fig. 1i.

Optical transformation of $\mathbf{A g}_{2} \mathrm{~S}$ dots into superdots. The structural changes induced in the $\mathrm{Ag}_{2} \mathrm{~S}$ dots by ultrafast laser pulses, summarized in Fig. 1, are accompanied by a dramatic change in their optical properties. This change is visible to the naked eye, as seen in Fig. 2a, which contains the optical images of a colloidal dispersion of as $\mathrm{Ag}_{2} \mathrm{~S}$ dots in $\mathrm{CHCl}_{3}$ before and after ultrafast laser irradiation ( $50 \mathrm{fs}, 90 \mathrm{~min}, 9 \mathrm{~W} \mathrm{~cm}^{-2}$ ). The dispersion changes color as a result of the ultrafast laser irradiation, becoming progressively more transparent (see Supplementary Fig. 6). Ultrafast laser irradiation reduces the absorbance of the sample in the $400-800 \mathrm{~nm}$ range by $35 \%$, as seen in Fig. $2 \mathrm{~b}$. This reduction occurs progressively during ultrafast laser irradiation, as shown in Supplementary Fig. 6. The absorption spectrum of the as-synthesized $\mathrm{Ag}_{2} \mathrm{~S}$ dots presents a shoulder around $400 \mathrm{~nm}$, which corresponds to the plasmonic band of sub-100-nm Ag
$\mathrm{NPs}^{36}$. This shoulder is no longer present after ultrafast laser irradiation. Besides modulating the optical absorption, ultrafast laser irradiation enhances the fluorescence brightness of the $\operatorname{Ag}_{2} \mathrm{~S}$ dots, as shown in the NIR-II fluorescence images in Fig. 2c. These correspond to a colloidal dispersion of $\mathrm{Ag}_{2} \mathrm{~S}$ dots before and after ultrafast laser irradiation ( $50 \mathrm{fs}, 90 \mathrm{~min}, 9 \mathrm{~W} \mathrm{~cm}^{-2}$ ). The emission of the as-synthesized $\mathrm{Ag}_{2} \mathrm{~S}$ dots is barely detectable with our NIRII imaging system, in contrast to the high signal observed after the irradiation process. This enhancement in the NIR-II brightness occurs progressively during ultrafast irradiation, as seen in Fig. 2d, which shows the time evolution of the NIR-II emission of $\mathrm{Ag}_{2} \mathrm{~S}$ dots during the process. The shape of the emission spectrum, as seen in the inset in Fig. 2d, remains virtually unchanged. This result indicates that the chemical nature of the fluorophore does not change upon irradiation. Fig. 2e shows the fluorescence QY of different colloidal dispersions of $\mathrm{Ag}_{2} \mathrm{~S}$ dots in $\mathrm{CHCl}_{3}$ after different durations of ultrafast laser irradiation $\left(50 \mathrm{fs}, 9 \mathrm{~W} \mathrm{~cm}^{-2}\right)$. Supplementary Fig. 7 includes some representative excitation and emission spectra used for QY calculations. The QY increases monotonously with the irradiation time for treatment durations shorter than $50 \mathrm{~min}$, while longer irradiations do not lead to any further increase. The QY of as-synthesized $\mathrm{Ag}_{2} \mathrm{~S}$ dots increases 
a

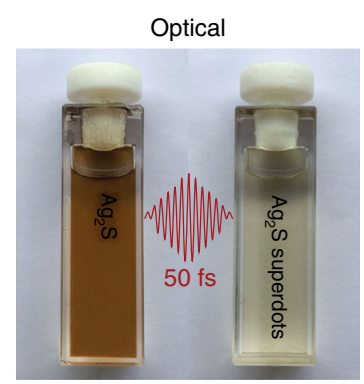

C

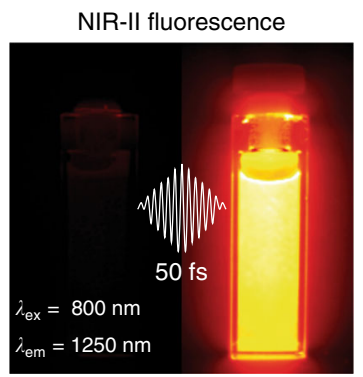

d
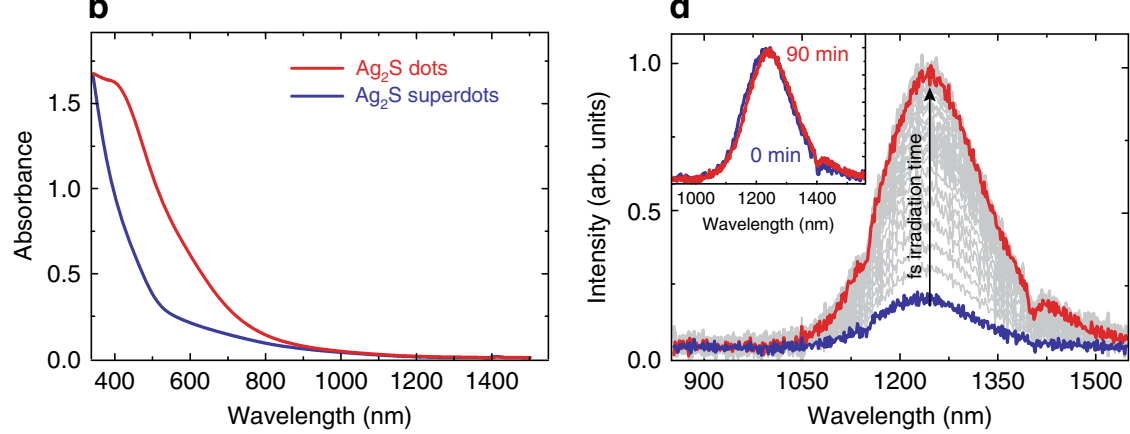
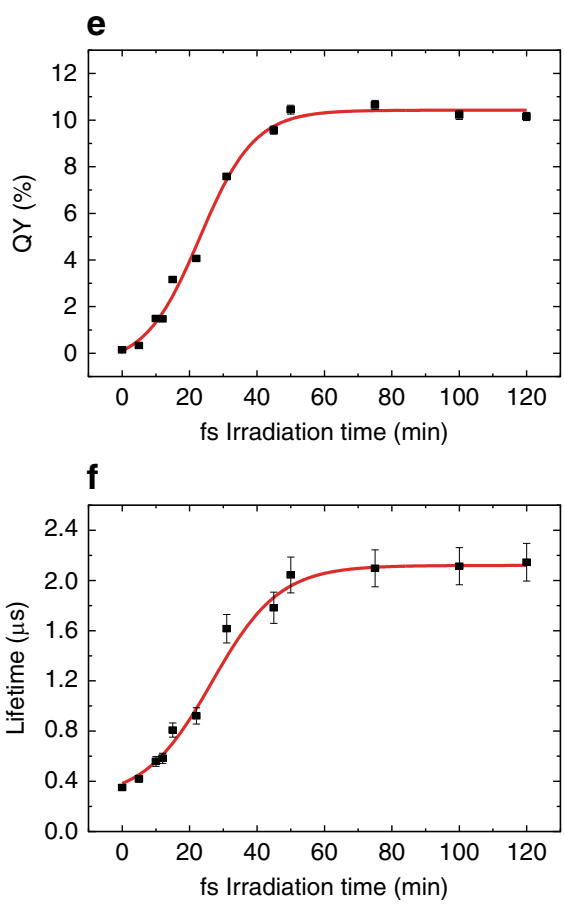

Fig. 2 Optical transformation of $\mathbf{A g}_{\mathbf{2}} \mathbf{S}$ dots into superdots. a Optical images, $\mathbf{b}$ UV-VIS-NIR extinction spectra, and $\mathbf{c}$ NIR-II fluorescence images of a colloidal dispersion of $\mathrm{Ag}_{2} \mathrm{~S}$ dots in $\mathrm{CHCl}_{3}$ before and after ultrafast laser irradiation ( $50 \mathrm{fs}, 90 \mathrm{~min}, 9 \mathrm{~W} \mathrm{~cm}^{-2}$ ). For the acquisition of NIR-II fluorescence images, the dispersions were optically excited with an $808 \mathrm{~nm}$ continuous wave laser diode $\left(100 \mathrm{~mW} \mathrm{~cm}^{-2}\right)$. d NIR-II emission spectra generated by a colloidal suspension of dots in $\mathrm{CHCl}_{3}$ during a 90 -min-long ultrafast laser irradiation. The inset shows the normalized emission spectra of the sample before and after the laser irradiation. e Fluorescence quantum yield and $\mathbf{f}$ fluorescence lifetime of $\mathrm{Ag}_{2} \mathrm{~S}$ dots in $\mathrm{CHCl}_{3}$ after being subjected to ultrafast laser irradiations processes of different durations. The error bars in $\mathbf{e}$ were calculated taking into account the equipment uncertainty that was determined from the statistical analysis of five measurements. In $\mathbf{f}$, each measurement was repeated five times and the error bars are the standard error of the mean of each series of measurements. Repetitive measurements to calculate both mean and error were performed on the same sample. The small relative uncertainties in QY (2\%) make the error bars too small to be visualized in $\mathbf{e . ~ I n ~} \mathbf{f}$, the error bars correspond to the standard error of the mean after measuring and analysing 10 decay curves for each sample. In $\mathbf{e}$ and $\mathbf{f}$, the squares correspond to the experimental data and the red lines are guides for the eyes.

from 0.13 to $10.7 \%$ in a 50 -min-long irradiation with 50 fs pulses. This constitutes an 80 -fold enhancement, validating our referring to the laser-irradiated $\mathrm{Ag}_{2} \mathrm{~S}$ dots as superdots. This QY enhancement is accompanied by a substantial increase in the fluorescence lifetime from $200 \mathrm{~ns}$ up to $2.1 \mu \mathrm{s}$, as shown in Fig. $2 \mathrm{f}$ (see Supplementary Fig. 6 for the decay curves). This lifetime increase follows the same trend with the irradiation time observed for the QY in Fig. 2e and is independent of the dot concentration in the $0.1-1 \mathrm{mg} \mathrm{mL}^{-1}$ range, as shown in Supplementary Fig. 8. These results rule out any possible contribution of fluorescence self-absorption to our lifetime measurements.

Mechanism of dot-to-superdot transformation. The dot-tosuperdot transformation induced by ultrafast laser irradiation depends critically on multiple experimental variables. Fig. 3a shows the time evolution of the NIR-II fluorescence intensity generated by a colloidal dispersion of dots in $\mathrm{CHCl}_{3}$ during ultrafast laser irradiation at different irradiation power densities for irradiation times up to $100 \mathrm{~min}$. The curves in Fig. 3a were obtained for constant pulse duration ( $50 \mathrm{fs})$, repetition rate $(1 \mathrm{kHz})$, and average power $(0.6 \mathrm{~W})$. For irradiation power densities below $3 \mathrm{~W} \mathrm{~cm}^{-2}$, which seems to be a threshold value, there is no apparent improvement in the luminescence properties. For irradiation power densities between 3 and $8 \mathrm{~W} \mathrm{~cm}^{-2}$, the NIR-II fluorescence intensity increases with the irradiation time. In this range of power densities, the slope of the intensity vs. time curve $(\eta=d I / d t)$ strongly depends on the irradiation pulse energy $\left(E_{p}\right)$. In fact, $\eta$ is proportional to $E_{p}^{2}$, which suggests that the dot-to-superdot transformation is triggered by a two-photon absorption process (see Supplementary Note 1 and Supplementary Fig. 9). For irradiation power densities between 8 and $9 \mathrm{~W} \mathrm{~cm}^{-2}$, the NIR-II fluorescence intensity increases with irradiation time until a stable value is reached. Finally, irradiation at or above $10 \mathrm{~W} \mathrm{~cm}^{-2}$ leads to a decrease in the emitted intensity for long irradiation times that may be attributed to sample degradation. This effect is highlighted for irradiation power densities close to $100 \mathrm{~W} \mathrm{~cm}^{-2}$. Under these conditions, an initial increase in the emission intensity is followed by an abrupt reduction, rendering a nonluminescent and completely transparent dispersion. Plotting the fluorescence intensity of the irradiated solution as a function of the irradiation power density evidences the existence of an optimum irradiation power density close to $9 \mathrm{~W} \mathrm{~cm}^{-2}$ (see Supplementary Fig. 10).

Fig. $3 \mathrm{~b}$ demonstrates that ultrafast laser-induced dot-tosuperdot transformation only occurs when the as-synthesized $\mathrm{Ag}_{2} \mathrm{~S}$ dots are dispersed in $\mathrm{CHCl}_{3}$. When they are dispersed in water, toluene, or hexane, pulsed irradiation leads to a decrease in their NIR-II fluorescence that can be attributed to laserinduced thermal loading (see Supplementary Note 2 and Supplementary Fig. 11). The presence of silver is also critical for the dot-to-superdot transformation. We corroborated this fact by analysing the effect of ultrafast laser irradiation in a $\mathrm{Ag}_{2} \mathrm{~S}$ dot dispersion free of $\mathrm{Ag}$ NPs (see Methods and Supplementary Fig. 12 for details). Ultrafast laser irradiation of these dots does not improve their fluorescence, as evidenced in Fig. 3c. Thus, ultrafast laser-driven dot-to-superdot transformation requires not only the presence of $\mathrm{CHCl}_{3}$ but also of Ag NPs in the dispersion. 

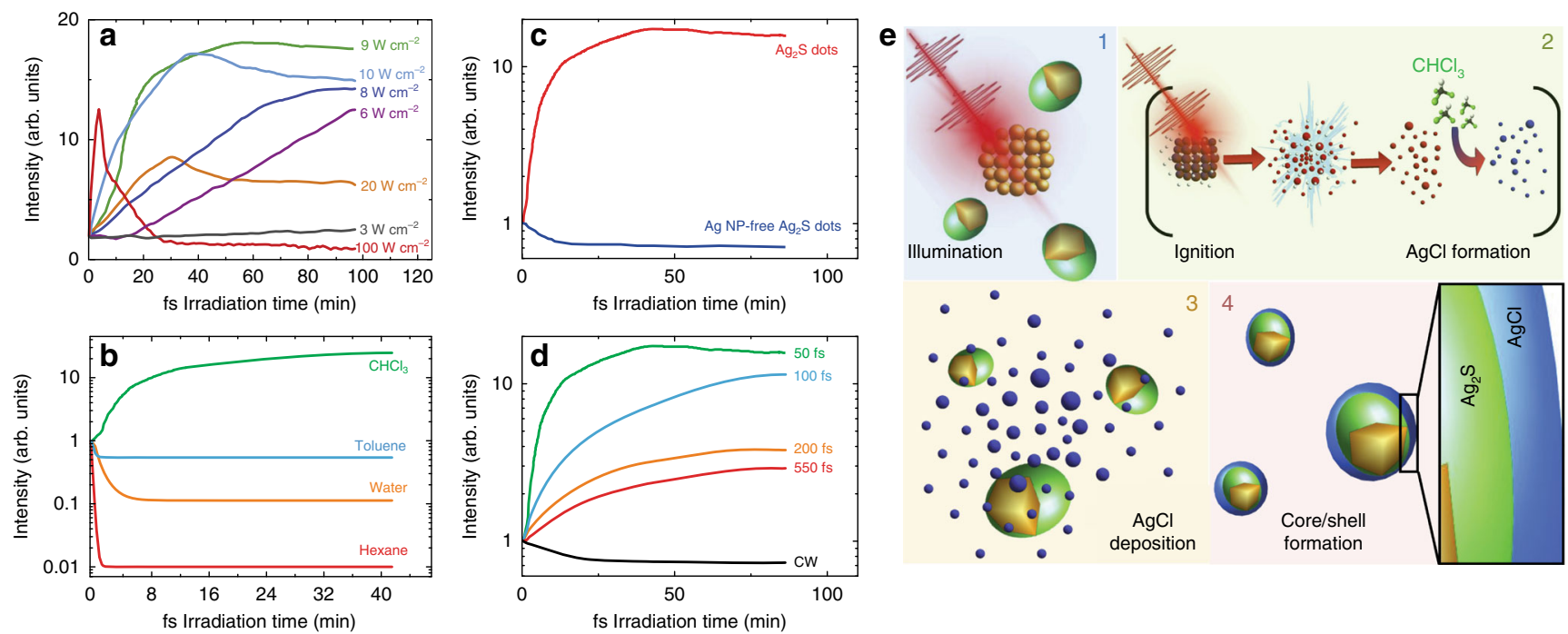

Fig. 3 Required conditions and mechanisms of laser-induced dot-to-superdot transformation. a Time evolution of the NIR-II fluorescence generated by colloidal dispersions of $\mathrm{Ag}_{2} \mathrm{~S}$ dots in $\mathrm{CHCl}_{3}$ under irradiation at different power densities. In all cases, pulse width and repetition rate were set to 50 fs and 1 $\mathrm{kHz}$, respectively. $\mathbf{b}$ Time evolution of the NIR-II fluorescence generated by $\mathrm{Ag}_{2} \mathrm{~S}$ dots dispersed in different solvents under laser irradiation with $50 \mathrm{fs}$, $808 \mathrm{~nm}$ laser pulses. c Time evolution of the NIR-II fluorescence generated by $\mathrm{Ag}_{2} \mathrm{~S}$ dots under laser irradiation with $50 \mathrm{fs}, 808 \mathrm{~nm}$ laser pulses in presence and absence of $\mathrm{Ag}$ NPs. $\mathbf{d}$ Time evolution of the NIR-II fluorescence intensity generated by $\mathrm{Ag}_{2} \mathrm{~S}$ dots in $\mathrm{CHCl}_{3}$ as obtained for different pulse durations. For all cases in $\mathbf{b}, \mathbf{c}$, and $\mathbf{d}$, the ultrafast laser power density was set to $9 \mathrm{~W} \mathrm{~cm}^{-2}$. Different samples were used in each condition used in $\mathbf{a}, \mathbf{b}, \mathbf{c}$ and $\mathbf{d}$. e Schematic representation of the physicochemical mechanisms underlying the ultrafast laser-induced dot-to-superdot transformation. Upon illumination with ultrafast infrared laser pulses, multiphoton excitation of Ag NPs leads to their Coulomb explosion (step 1). The $\mathrm{Ag}^{+\mathrm{z}}$ generated in this process react with $\mathrm{CHCl}_{3}$ molecules forming $\mathrm{AgCl}$ (step 2), which in turn reacts with the surface of the $\mathrm{Ag}_{2} \mathrm{~S}$ dots forming a protective layer (steps 3 and 4 ).

Moreover, the dynamics of the dot-to-superdot transformation depends critically on the duration of the laser pulses. Fig. 3d shows the time evolution of the NIR-II intensity emitted by a dispersion of $\mathrm{Ag}_{2} \mathrm{~S}$ dots in $\mathrm{CHCl}_{3}$ during irradiation with laser pulses of different durations and identical power $(0.6 \mathrm{~W})$ and power density $\left(9 \mathrm{~W} \mathrm{~cm}^{-2}\right)$. The efficiency of the dot-to-superdot transformation, estimated from the magnitude of the enhancement in the NIR-II emission, decreases for longer pulse durations. Irradiation with a continuous wave laser does not improve the NIR-II fluorescence brightness, indicating that no dot-tosuperdot transformation occurs.

The experimental results shown in Figs. 1-3 allow us to provide a plausible explanation for the dot-to-superdot transformation. This is schematically shown in Fig. 3e. Due to the synthesis route used here, the as-prepared dispersions of $\mathrm{Ag}_{2} \mathrm{~S}$ dots also contain $\mathrm{Ag}$ NPs. The plasmon resonance of these $\mathrm{Ag}$ particles is responsible for the extinction peak observed at around $400 \mathrm{~nm}$ (see Fig. 2b). When excited with $808 \mathrm{~nm}$ ultrafast pulses, twophoton absorption by Ag NPs induces a high free electron density that results in the Coulomb explosion of the Ag NPs (step 1 in Fig. 3e $)^{37}$. The key role of multiphoton excitation is supported by the requirement of ultrafast laser pulses that ensure high photon densities. These allow for sequential absorption through virtual states of $\mathrm{Ag} \mathrm{NPs}^{38}$. The participation of two $808 \mathrm{~nm}$ photons in this process is further supported by the experimental data in Supplementary Fig. 9. The Coulomb explosion of Ag NPs leads to an increment in the concentration of highly reactive $\mathrm{Ag}^{+}$in the solution, which react with $\mathrm{CHCl}_{3}$ yielding silver chloride $(\mathrm{AgCl}$, step 2 in Fig. 3e). The laser-generated $\mathrm{AgCl}$ molecules interact with the surface of the $\mathrm{Ag}_{2} \mathrm{~S}$ dots, where a protective a $\mathrm{AgCl}$ shell is formed (steps 3 and 4 in Fig. 3e). Therefore, after ultrafast laser irradiation, the low-bandgap semiconductor $\mathrm{Ag}_{2} \mathrm{~S}(0.9 \mathrm{eV})$ is coated with an inorganic shell of higher-bandgap $\mathrm{AgCl}(5.13 \mathrm{eV})$ several monolayers thick, as seen in Fig. $1 \mathrm{i}^{39}$. This protective shell strongly reduces the non-radiative transitions that involve the vibronic activation of solvent $\mathrm{CHCl}_{3}$ molecules and prevents the formation of shallow or deep midgap states as surface traps that would provide non-radiative deexcitation pathways. The reduction in the non-radiative decay probabilities caused by this protective shell simultaneously explains the increment in the NIR-II QY and the fluorescence lifetime. The decrease in the non-radiative decay probability is also evidenced by a reduction in the light-to-heat conversion efficiency when the dot-to-superdot transformation takes place (see Supplementary Fig. 13 and Supplementary Note 3). Both dots and superdots can be damaged under excessive irradiation doses, explaining why at high irradiation power densities the initial enhancement in NIR-II luminescence is followed by a nonreversible quenching (see Fig. 3a). An arising question is why the multiphoton excitation and subsequent Coulomb explosion only occur in the Ag NPs and not in the Ag cores within the $\mathrm{Ag}_{2} \mathrm{~S}$ dots. A plausible explanation is that $\mathrm{Ag}$ cores have no plasmonic resonance that can be excited by $808 \mathrm{~nm}$ fs laser pulses due to being surrounded by $\mathrm{Ag}_{2} \mathrm{~S}$. The very different dielectric constants/refractive indices of $\mathrm{CHCl}_{3}$ and $\mathrm{Ag}_{2} \mathrm{~S}$ could be behind this effect, since the spectral location of the plasmon resonance of Ag NPs strongly depends on the dielectric constant/ refractive index of the surrounding medium ${ }^{40}$. The absence of multiphoton excitation of the Ag core would prevent its Coulomb explosion, required for the formation of the $\mathrm{AgCl}$ shell ${ }^{41}$.

In vivo NIR-II imaging with $\mathbf{A g}_{2} \mathrm{~S}$ superdots. To test the potential application of $\mathrm{Ag}_{2} \mathrm{~S}$ superdots for in vivo NIR-II imaging, we transferred them from their original solvent $\left(\mathrm{CHCl}_{3}\right)$ to phosphate buffer saline (PBS) by means of a ligand exchange procedure described in Methods. The adhesion of hydrophilic and bifunctional HS-PEG-COOH ligands on the surface of $\mathrm{Ag}_{2} \mathrm{~S}$ superdots makes them stable in PBS with no signs of precipitation for at least 12 months. The average hydrodynamic diameter and Z-potential after ligand exchange are $22 \mathrm{~nm}$, and $-25 \mathrm{mV}$, respectively. PEG coating and dispersion in PBS does not significantly reduce the fluorescence 
a

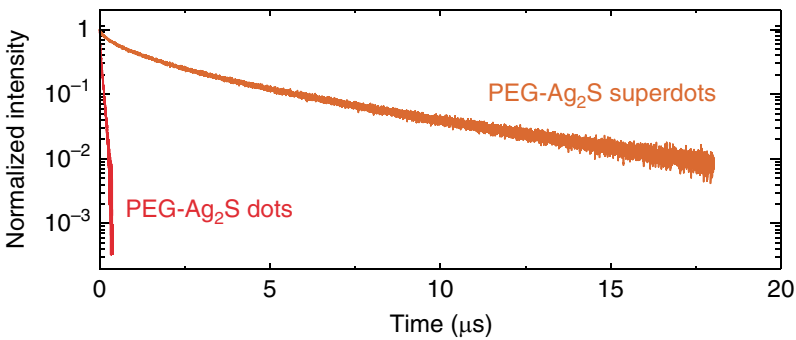

C

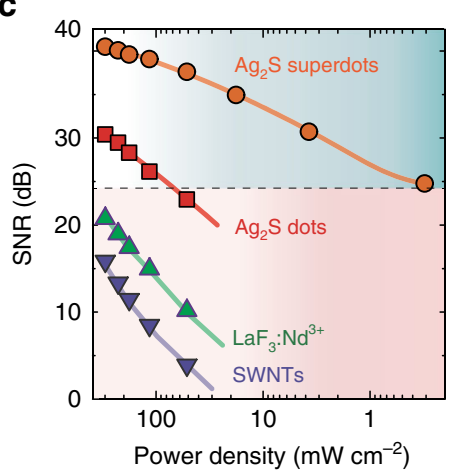

d
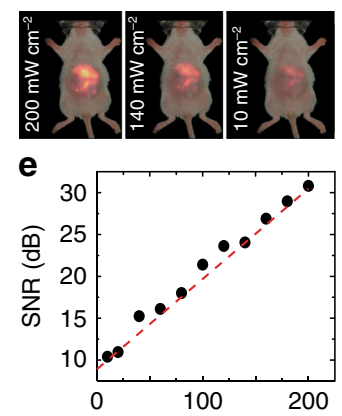

Power density $\left(\mathrm{mW} \mathrm{cm}^{-2}\right)$ b

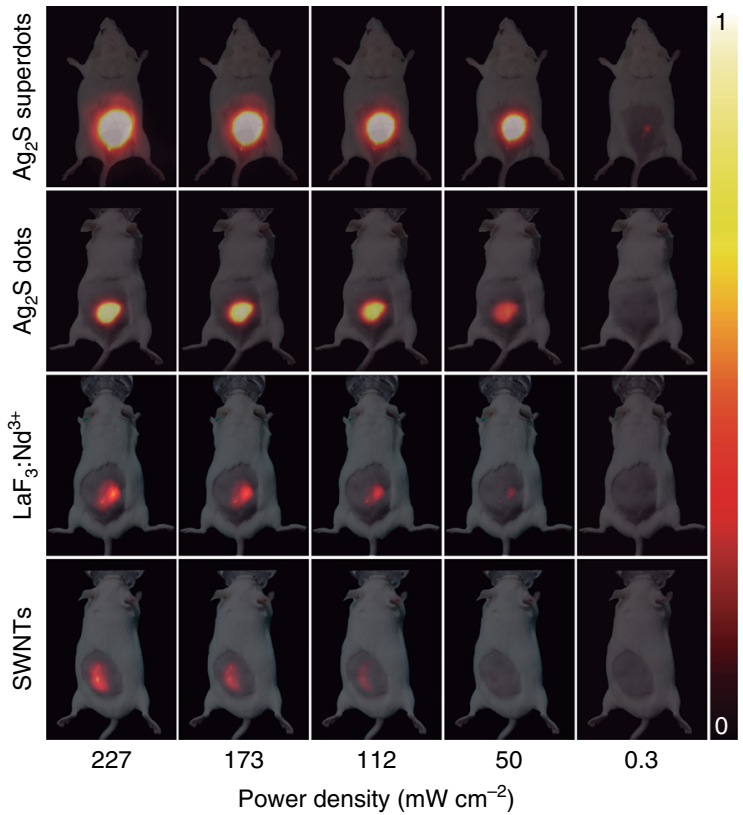

f

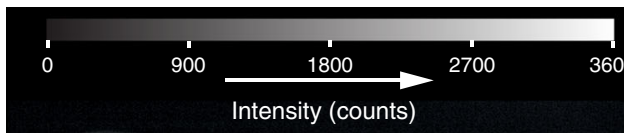

g

h

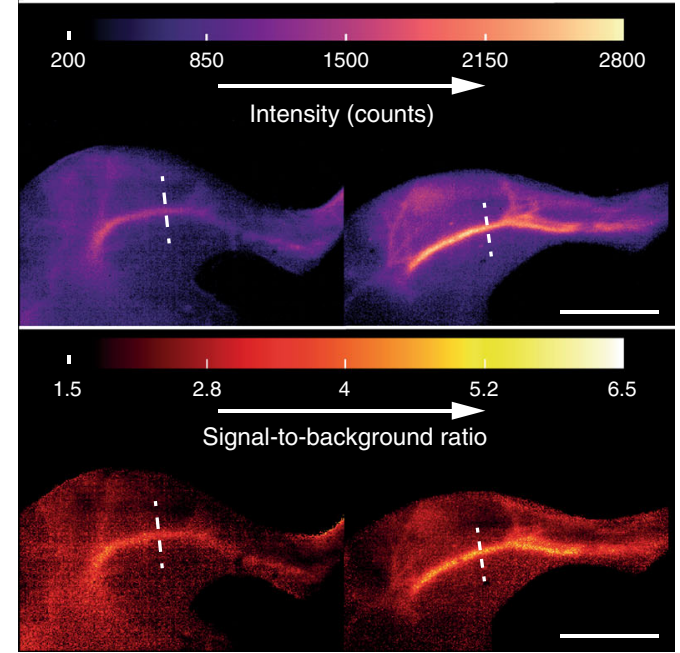

decay time of $\mathrm{Ag}_{2} \mathrm{~S}$ superdots, which is close to $2 \mu$ s (see Fig. 4a). The fact that $\mathrm{Ag}_{2} \mathrm{~S}$ superdots show the same decay time in $\mathrm{CHCl}_{3}$ and $\mathrm{PBS}$ reveals the effectiveness of the $\mathrm{AgCl}$ protective shell against the appearance of multiphonon relaxation events favored by the vibration modes of water molecules. This, in turn, results in identical QY values $(\approx 10 \%)$ in both solvents. Fig. $4 \mathrm{a}$ also includes, for the sake of comparison, the

\section{$\mathbf{i}$}

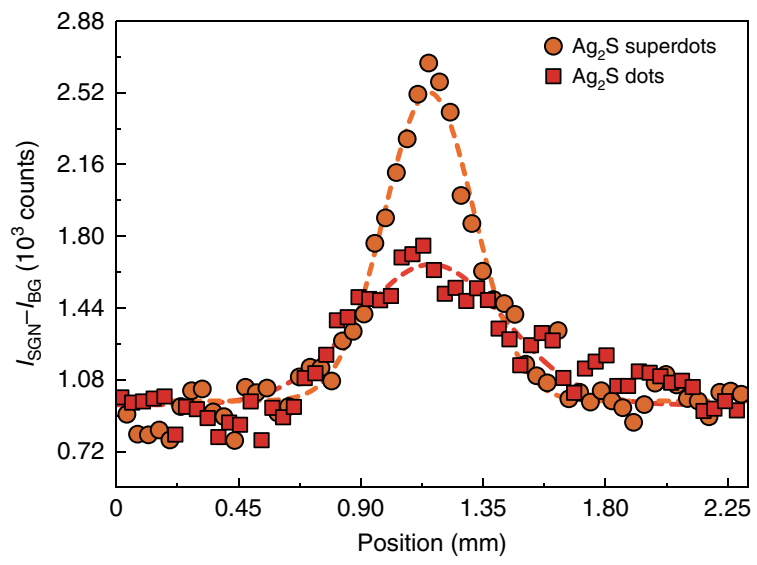

j

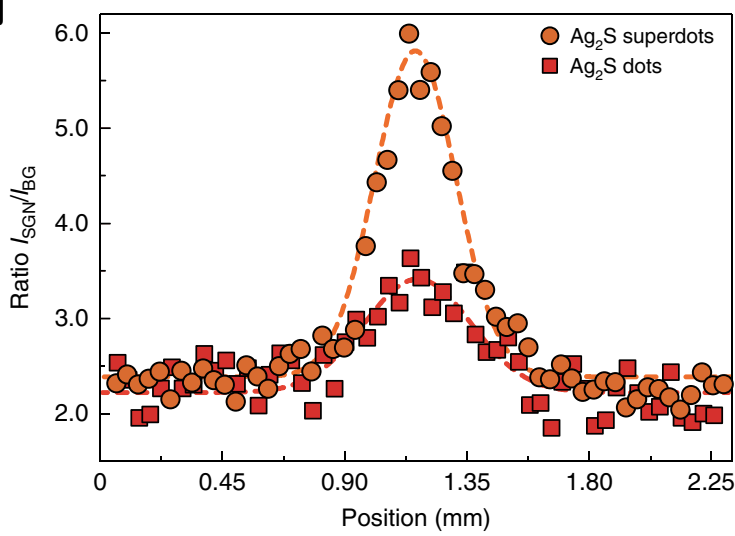

fluorescence decay curve obtained from commercially available (Sinano Corp. China) PEG-coated $\mathrm{Ag}_{2} \mathrm{~S}$ dots dispersed in PBS. These show a fluorescence lifetime of $53 \mathrm{~ns}$, about 40 times shorter than that of PBS-dispersed PEG-coated $\mathrm{Ag}_{2} \mathrm{~S}$ superdots. Such a long fluorescence lifetime and high QY suggests the potential application of PEGylated $\mathrm{Ag}_{2} \mathrm{~S}$ superdots for highcontrast, low-dose NIR-II in vivo imaging. 
Fig. 4 In vivo brightness of $\mathbf{A g}_{\mathbf{2}} \mathbf{S}$ superdots: a comparison with their competitors. a NIR-II fluorescence decay curves of PEG-coated $A g_{2} S$ dots (provided by Sinano Corp., China) and $\mathrm{Ag}_{2} \mathrm{~S}$ superdots, both dispersed in PBS. b NIR-II fluorescence images of a group of four mice subcutaneously injected with colloidal aqueous dispersions containing $\mathrm{Ag}_{2} \mathrm{~S}$ superdots, commercial $\mathrm{Ag}_{2} \mathrm{~S}$ dots, SWNTs, and $\mathrm{LaF}_{3}: \mathrm{Nd} \mathrm{NPs}$. The same NP volume (100 $\mu \mathrm{L}$ ) and concentration $\left(1.5 \mathrm{mg} \mathrm{mL}^{-1}\right)$ were injected in all cases. The different images for same optical probe correspond to different $808 \mathrm{~nm}$ illumination power densities. c Signal-to-noise ratio (SNR) as a function of power density quantified from the analysis of images in $\mathbf{b}$ for the four NIR-II nanoprobes evaluated. d Fluorescence images of $\mathrm{Ag}_{2} \mathrm{~S}$ superdots accumulated in the liver obtained at three different $808 \mathrm{~nm}$ excitation power densities. e Power density dependence of SNR calculated from in vivo NIR-II fluorescence images at different excitation power densities. $\mathbf{f}$ NIR-II fluorescence images of the left hind limbs of two mice immediately before (top) and $15 \mathrm{~s}$ after (bottom) of an intravenous injection of $\mathrm{Ag}_{2} \mathrm{~S}$ dots (left) or superdots (right). $\mathbf{g}$ Net intensity images obtained from subtracting the background images (top row in $\mathbf{f}$ ) from the signal images (bottom row in $\mathbf{f}$ ). $\mathbf{h}$ Signal-to-background images obtained by dividing the signal intensity images (bottom row in $\mathbf{f}$ ) by the background images (top row in $\mathbf{f}$ ). Scale bars in $\mathbf{f}, \mathbf{g}$, and $\mathbf{h}$ are $2 \mathrm{~mm}$. i Net intensity and $\mathbf{j}$ signal-to-background ratio obtained along a line profile across the saphenous artery (indicated as dashed white lines in $\mathbf{g}$ and $\mathbf{h}$ ).

To evaluate the performance of $\mathrm{Ag}_{2} \mathrm{~S}$ superdots in in vivo imaging, we benchmarked them against other well-established NIR-II fluorescent probes: commercial $\mathrm{Ag}_{2} \mathrm{~S}$ dots, neodymiumdoped nanocrystals $\left(\mathrm{LaF}_{3}: \mathrm{Nd}^{3+}\right)$, and single-walled carbon nanotubes (SWNTs) ${ }^{42-44}$. FDA-approved indocyanine green was not included in this comparison as preliminary experiments revealed the poor spectral overlap between its emission tail and the spectral response of our NIR-II imaging system (see Supplementary Figs. 14 and 15) ${ }^{45,46}$. For our comparative study, we used four different mice that were subcutaneously injected with $100 \mu \mathrm{L}$ of a PBS solution containing, in each case, $\mathrm{Ag}_{2} \mathrm{~S}$ superdots, $\mathrm{Ag}_{2} \mathrm{~S}$ dots, $\mathrm{LaF}_{3}: \mathrm{Nd}^{3+}$, and SWNTs. The NP concentration was set in all cases to $1.5 \mathrm{mg} \mathrm{mL}^{-1}$, corresponding to a total injected NP dose of $\approx 5 \mathrm{mg} \mathrm{kg}^{-1}$. Fig. $4 \mathrm{~b}$ shows the in vivo NIR-II fluorescence images obtained in each case for 808 $\mathrm{nm}$ illumination power densities ranging from 227 down to 0.3 $\mathrm{mW} \mathrm{cm}{ }^{-2}$. These results indicate that $\mathrm{Ag}_{2} \mathrm{~S}$ superdots outperform all other NIR-II fluorescent probes evaluated here from a brightness standpoint (see Supplementary Table 1). We considered the possibility of experimental error in these results due to individual variations in the thickness of the mouse skin. However, this would only account for $20 \%$ of the total variation in the transmitted fluorescence $( \pm 80 \mu \mathrm{m}$ over a $400 \mu \mathrm{m}$ layer of skin, see Supplementary Note 4 ). These variations are negligible compared with the dramatic difference in the emission intensity observed experimentally between the $\mathrm{Ag}_{2} \mathrm{~S}$ superdots and the rest of the evaluated fluorescent probes. The superior brightness of the superdots enables the acquisition of fluorescence images with sufficient contrast even at the ultra-low irradiation power density of $0.3 \mathrm{~mW} \mathrm{~cm}^{-2}$, where no fluorescence from any of the other three tested NIR-II probes could be registered. This not only allows in vivo imaging with cost-effective excitation sources but also ensures a negligible thermal loading during image acquisition (see Supplementary Fig. 16) (38 $^{38}$ The superior contrast achieved imaging with $\mathrm{Ag}_{2} \mathrm{~S}$ superdots is quantified in Fig. 4c, which shows the power density dependence of the signal-to-noise ratio (SNR) calculated from the fluorescence images in Fig. 4b. From Fig. 4c, we conclude that the brightness improvement achieved during ultrafast laser irradiation reduces the minimum illumination density that can be used for in vivo imaging by almost two orders of magnitude. This enhancement is also evident when comparing the illumination conditions used in this work to those reported previously for $\mathrm{Ag}_{2} \mathrm{~S}$ dots, SWNTs, and lanthanide-doped nanocrystals (see Supplementary Table 2).

The data in Fig. 4c correspond to the analysis of in vivo images of subcutaneous injections. To provide a realistic figure of the minimum power density required for in vivo imaging, we performed similar experiments for intravenously injected $\mathrm{Ag}_{2} \mathrm{~S}$ superdots. Injection volume and concentration were set to $100 \mu \mathrm{L}$ and $0.15 \mathrm{mg} \mathrm{mL}^{-1}$, respectively. Fig. $4 \mathrm{~d}$ shows the in vivo fluorescence images for three different excitation power densities obtained $30 \mathrm{~min}$ after intravenous injection of a dispersion of $\mathrm{Ag}_{2} \mathrm{~S}$ superdots. At this time point, the superdots had accumulated mostly in the liver and the spleen. Fig. 4e shows the linear dependence of the SNR with the power density, indicating that even at a very low excitation intensity $\left(10 \mathrm{~mW} \mathrm{~cm}^{-2}\right)$ the SNR is above $10 \mathrm{~dB}$. This is a major improvement with respect to the intensities required for in vivo imaging with $\mathrm{Ag}_{2} \mathrm{~S}$ dots (see Supplementary Table 2), which are typically above $100 \mathrm{~mW} \mathrm{~cm}^{-2}$. The high brightness of $\mathrm{Ag}_{2} \mathrm{~S}$ superdots also allows imaging at large depths $(>1 \mathrm{~cm})$ into tissues (see Supplementary Note 5 and Supplementary Fig. 17).

$\mathrm{Ag}_{2} \mathrm{~S}$ superdots enable improved in vivo visualization of blood vessels, as shown in Fig. $4 \mathrm{f}-\mathrm{j}$. The images in Fig. $4 \mathrm{f}-\mathrm{h}$ correspond to the left hind limbs of two mice after intravenous injection of either $\mathrm{Ag}_{2} \mathrm{~S}$ dots or superdots (see Methods). Injection volume and concentration were set to $100 \mu \mathrm{L}$ and $0.15 \mathrm{mg} \mathrm{mL}^{-1}$, respectively. Fig. $4 \mathrm{f}$ shows the magnified luminescence images of the limb immediately before (top) and $15 \mathrm{~s}$ after injection (bottom). Although the preinjection background (caused by tissue autofluorescence) was similar in both cases, the image contrast after injection is substantially higher after injection of superdots (right) than in the case of dots (left). We quantified the improvement in image contrast by calculating the signal-tobackground ratio for both sets of images. This was done by subtracting the background from the overall emission to obtain net signal images (Fig. $4 \mathrm{~g}$ ), which were also divided by the background to calculate the signal-to-background ratios (Fig. 4h). Both the net signal $\left(\mathrm{I}_{\mathrm{SG}}-\mathrm{I}_{\mathrm{BG}}\right.$, Fig. $\left.4 \mathrm{i}\right)$ and the signal-tobackground $\left(\mathrm{I}_{\mathrm{SG}} / \mathrm{I}_{\mathrm{BG}}\right.$, Fig. $4 \mathrm{j}$ ) line profiles along the saphenous artery (dashed lines in Fig. 4g, h), indicated a superior performance of the $\mathrm{Ag}_{2} \mathrm{~S}$ superdots as compared with dots. The pixel profiles in Fig. $4 \mathrm{i}$, $\mathrm{j}$ indicate that $\mathrm{Ag}_{2} \mathrm{~S}$ superdots improve the net signal by $60 \%$ and the signal-to-background ratio by $90 \%$ when compared with conventional $\mathrm{Ag}_{2} \mathrm{~S}$ dots. Further discussion on the NIR-II images, focusing on the relative quality of the ones presented here to those previously reported by other groups ${ }^{15,16,18,29,31,47}$, is included in Supplementary Note 6, Supplementary Fig. 18 and Supplementary Table 3.

$\mathrm{Ag}_{2} \mathrm{~S}$ superdots also outperform conventional $\mathrm{Ag}_{2} \mathrm{~S}$ dots at in vivo video rate NIR-II fluorescence imaging. Fig. 5a shows the NIR-II images obtained at different times after intravenous administration of PEG-coated $\mathrm{Ag}_{2} \mathrm{~S}$ superdots $\left(100 \mu \mathrm{L}, 0.15 \mathrm{mg} \mathrm{mL}^{-1}\right)$. The total dose $\left(15 \mu \mathrm{g}, \sim 0.5 \mathrm{mg} \mathrm{kg}^{-1}\right)$ is more than one order of magnitude smaller than the administered dose $\left(6.6 \mathrm{mg} \mathrm{kg}^{-1}\right)$ reported for NIRII video rate recording with $\mathrm{Ag}_{2} \mathrm{~S}$ dots ${ }^{29}$. The use of low administration doses for in vivo imaging is beneficial for different reasons. First, to develop a cost-effective probe for NIR-II in vivo imaging, the amount of material required to achieve high contrast in vivo images should be reduced. Low administration doses minimize potential toxicity of the probe. Further, $\mathrm{Ag}_{2} \mathrm{~S}$ superdots show some photothermal effect that requires low administration doses and illumination intensities to prevent undesirable heating during in vivo imaging. For the conditions used in this work, 

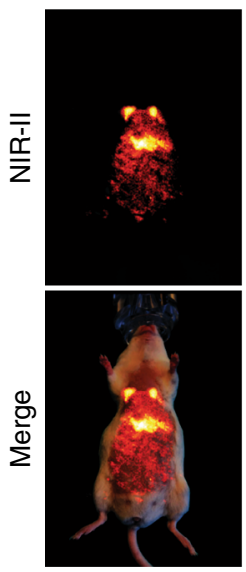

$3 \min$
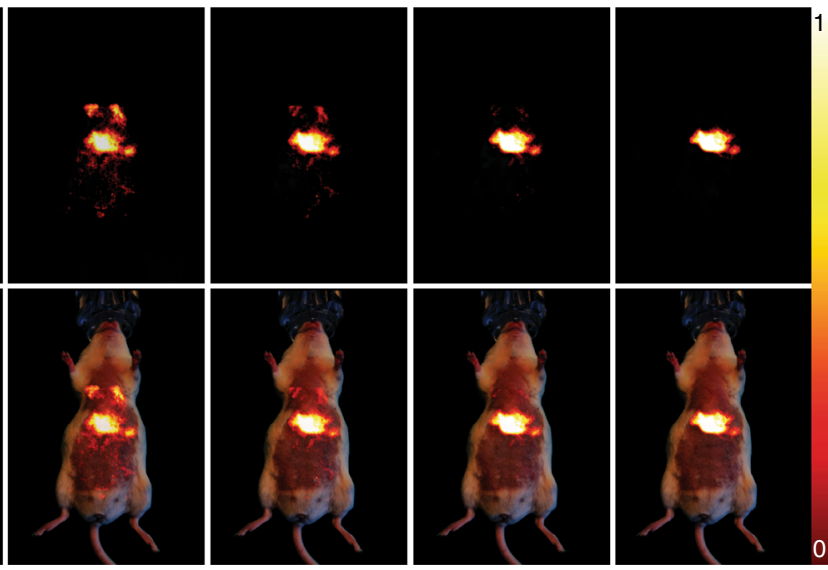

$10 \min$

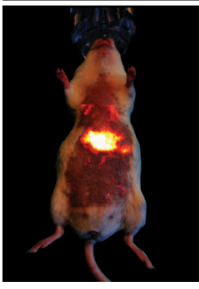

$30 \min$

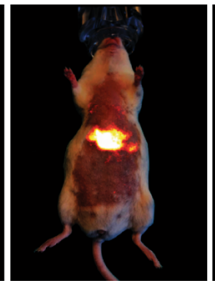

$70 \mathrm{~min}$

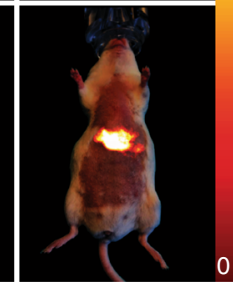

$100 \min$
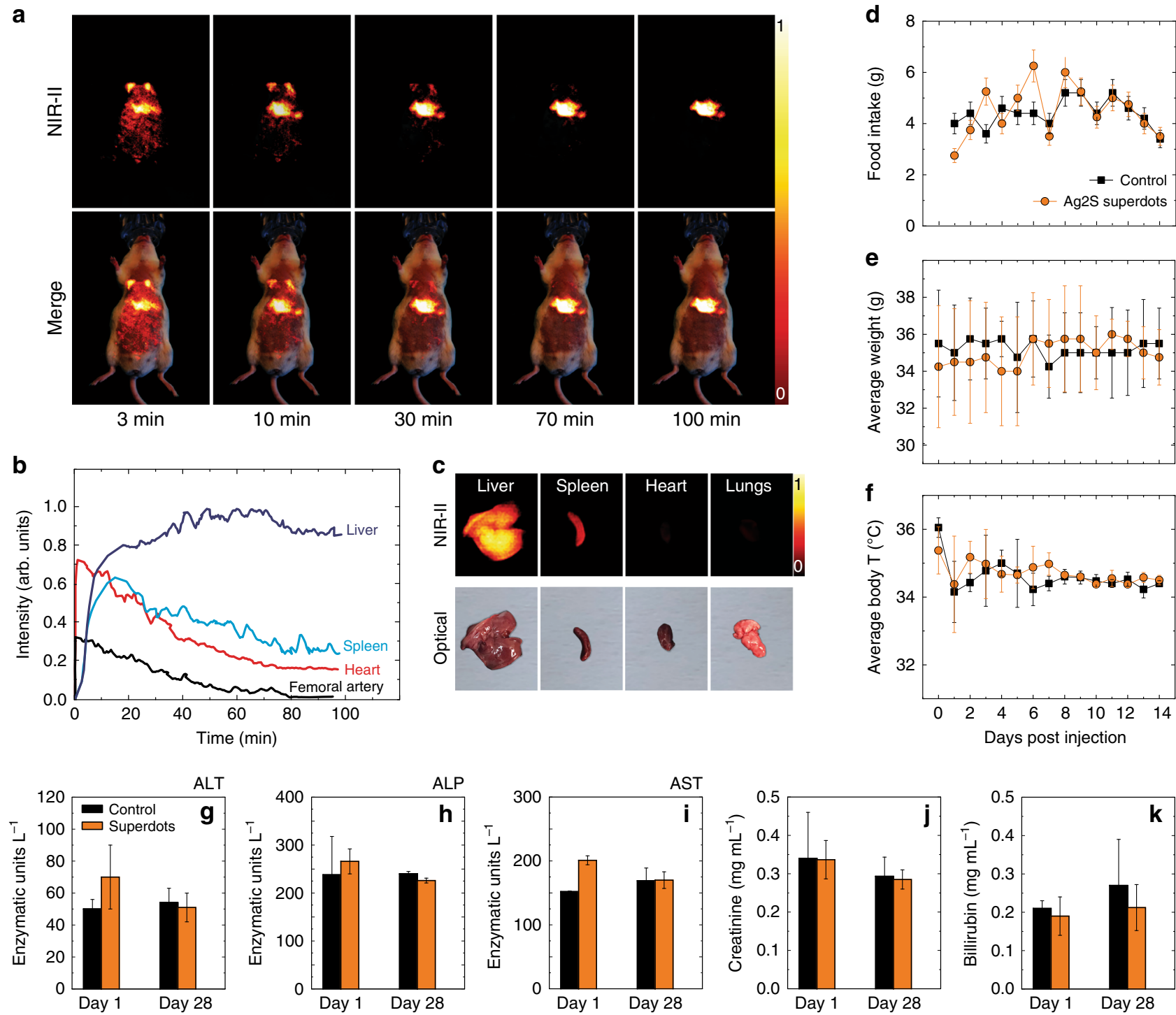

Fig. 5 In vivo time-resolved imaging with $\mathbf{A g}_{\mathbf{2}} \mathbf{S}$ superdots. a NIR-II fluorescence images obtained at different times after intravenous injection of $\mathrm{Ag}_{2} \mathrm{~S}$ superdots dispersed in PBS $\left(100 \mu \mathrm{L}, 0.15 \mathrm{mg} \mathrm{mL}^{-1}\right)$. b Time evolution of the fluorescence intensity at the liver, spleen, heart, and femoral artery after intravenous injection of $\mathrm{Ag}_{2} \mathrm{~S}$ superdots. c NIR-II ex vivo fluorescence and optical images of the liver, spleen, heart, and lungs obtained from a mouse euthanized $100 \mathrm{~min}$ after intravenous injection of $\mathrm{Ag}_{2} \mathrm{~S}$ superdots. Time evolution of daily food intake (d), weight (e), and body temperature (f) of four CD1 mice after intravenous injection of $300 \mu \mathrm{L}$ of a dispersion of $\mathrm{Ag}_{2} \mathrm{~S}$ superdots in PBS ( $0.5 \mathrm{mg} \mathrm{mL} \mathrm{L}^{-1}$, total dose of $\left.150 \mu \mathrm{g}\right)$. The results obtained from a control group intravenously injected with $300 \mu \mathrm{L}$ of PBS are included for comparison. Serum concentration $(\mathbf{g})$ of hepatic enzymes ( $\mathbf{g}$, $\mathbf{h}$ and $\mathbf{i})$ for mice intravenously injected with $\mathrm{Ag}_{2} \mathrm{~S}$ superdots and control mice as obtained 1 and 28 days after injection. Serum concentration of creatinine ( $\mathbf{j}$ ) and bilirubin (k) corresponding to mice subjected to an intravenous injection of $\mathrm{Ag}_{2} \mathrm{~S}$ superdots and control mice as obtained 1 and 28 days after injection. ( $n=3$ for each group). The error bars correspond to the standard error of the mean ( \pm SEM).

there is negligible thermal loading due to the photothermal conversion of $\mathrm{Ag}_{2} \mathrm{~S}$ superdots (see Supplementary Fig. 19). The images shown in Fig. 5a were obtained under $808 \mathrm{~nm}$ excitation at $10 \mathrm{~mW} \mathrm{~cm} \mathrm{~cm}^{-2}$, which is one order of magnitude lower than the illumination power density reported for NIR-II video recording using SWNTs $\left(140 \mathrm{~mW} \mathrm{~cm}^{-2}\right)^{43}$. The illumination power density here used is also much lower than the safety threshold at this wavelength $\left(329 \mathrm{~mW} \mathrm{~cm}^{-2}\right)$ established by the International Commission on Non-ionizing Radiation Protection (ANSI Z136.1-2000) ${ }^{48}$. NIR-II video recording enabled us to track the in vivo biodistribution of our $\mathrm{Ag}_{2} \mathrm{~S}$ superdots. The time evolution of the NIR-II fluorescence intensity generated by the $\mathrm{Ag}_{2} \mathrm{~S}$ superdots at the liver, spleen, heart, and femoral artery after intravenous injection is shown in Fig. 5b. During the first 3 min after injection, the PEG-coated superdots were circulating and gathering in the liver, spleen, and heart. The fluorescence signal observed at the femoral artery is assigned to the presence of $\mathrm{Ag}_{2} \mathrm{~S}$ superdots in the bloodstream. Fitting this curve to a first-order exponential, we estimate a blood half-life close to $20 \mathrm{~min}$ for the $\mathrm{Ag}_{2} \mathrm{~S}$ superdots. As seen in Fig. 5a, b, most of the superdots have been uptaken by the liver and spleen after $30 \mathrm{~min}$. This is supported by ex vivo NIR-II images taken 100 min after injection (Fig. 5c). The accumulation of NPs in the liver and spleen has been widely reported and is related to filtration mechanisms promoted by the reticuloendothelial system ${ }^{49}$.

To evaluate the in vivo biocompatibility of our $\mathrm{Ag}_{2} \mathrm{~S}$ superdots, we analysed the time evolution of weight, food intake, and body temperature of four CD1 mice for 2 weeks after intravenous administration of $\mathrm{Ag}_{2} \mathrm{~S}$ superdots dispersed in PBS. The injected dose $\left(150 \mu \mathrm{g}\right.$, equivalent to $\left.5 \mathrm{mg} \mathrm{kg}^{-1}\right)$ is one order of magnitude 
above that used for NIR-II in vivo video recording. Four additional mice were injected with $300 \mu \mathrm{L}$ of PBS and used as a control. Experimental data included in Fig. $5 \mathrm{~d}-\mathrm{f}$ reveal that, even at such relatively high administration doses, there are no relevant differences between both groups. These findings are consistent with the reported low toxicity of conventional $\mathrm{Ag}_{2} \mathrm{~S}$ dots and with the already demonstrated ability of $\mathrm{AgCl}$ coatings to minimize $\mathrm{NP}$ cytotoxicity $^{50,51}$. To further test the long-term biocompatibility of $\mathrm{Ag}_{2} \mathrm{~S}$ superdots, we performed a 28-day subchronic toxicological experiment and histological analyses, as detailed in the Supplementary Methods. Supplementary Figs. 20 and 21 show the time course of ALT, ALP and AST hepatic enzymes, creatinine and bilirubin for mice injected with $\mathrm{Ag}_{2} \mathrm{~S}$ superdots $\left(100 \mu \mathrm{L}, 0.15 \mathrm{mg} \mathrm{mL}^{-1}\right)$. The serum concentrations of all biomarkers lie within healthy ranges. The values obtained 1 and 28 days after injection are shown in Fig. $5 \mathrm{~g}-\mathrm{k}$ both for mice intravenously injected with $\mathrm{Ag}_{2} \mathrm{~S}$ superdots and for control mice. The hepatic enzymes show a discrete though not relevant increase at acute time points $(24 \mathrm{~h})$, while creatinine and bilirubin remain unaffected. Twenty-eight days after injection, the levels of all five metabolites are comparable between both groups. A more complete discussion of the time-course evolution of all the biomarkers is included in Supplementary Note 7. These results suggest a low in vivo toxicity for the $\mathrm{Ag}_{2} \mathrm{~S}$ superdots, which is consistent with the previously reported good biocompatibility of $\mathrm{Ag}_{2} \mathrm{~S}$ dots ${ }^{41}$. This is further supported by the low cytotoxicity of $\mathrm{Ag}_{2} \mathrm{~S}$ superdots (see Supplementary Fig. 22) and in histological assays (Supplementary Fig. 23). This is a promising result, although it is important to remark that the clinical translation of $\mathrm{Ag}_{2} \mathrm{~S}$ superdots requires further toxicity experiments including studies of the maximum tolerated dose, the clearance pathways and the effect of $\mathrm{Ag}_{2} \mathrm{~S}$ superdots on physiology, metabolism, behavior, and cognition. Thus, such translation is not immediate.

\section{Discussion}

Although $\mathrm{Ag}_{2} \mathrm{~S}$ dots have shown excellent properties as NIR-II optical probes their potential use in preclinical applications is limited by their low brightness. Traditional synthesis routes lead to $\mathrm{Ag}_{2} \mathrm{~S}$ dots with QY typically below $1 \%$. Femtosecond laser pulses can enhance this QY by more than one order of magnitude, up to values above 10\% (Fig. 2e), without affecting the spectral shape of the broadband emission centered at $1200 \mathrm{~nm}$ (Fig. 2d).

The dot-to-superdot transformation triggered by femtosecond laser pulses is strongly dependent on the experimental conditions. It requires the presence of silver NPs and chloroform molecules (Fig. 3b, c). Pulse widths longer than $100 \mathrm{fs}$ lead to slow and inefficient dot-to-superdot transformation (Fig. 3d). We concluded that dot-to-superdot transformation is a sequential process (Fig. 3e): (i) femtosecond laser pulses induce the Coulomb explosion of silver NPs, (ii) silver atoms react with solvent molecules, and (iii) a protective shell of $\mathrm{AgCl}$ surrounds $\mathrm{Ag}_{2} \mathrm{~S}$ dots. This protective shell (evidenced by electron microscopy, Fig. 1) drastically reduces surface-related non-radiative processes and leads to an increased brightness and a longer fluorescence lifetime (Fig. 2f).

In vivo NIR-II imaging experiments demonstrated the ability of $\mathrm{Ag}_{2} \mathrm{~S}$ superdots for low-dose (administration and irradiation) preclinical imaging (Fig. 4b, c). We demonstrated how the optimization of the optical properties caused by femtosecond laser pulses improves both contrast and resolution of deep tissue in vivo images (Fig. 4g, h). An array of in vitro toxicity, biochemical and histological assays suggested a low toxicity for the $\mathrm{Ag}_{2} \mathrm{~S}$ superdots (Fig. 5).

Our discovery, the ability of ultrafast laser pulses to improve the luminescence properties of NPs, also stimulates the development of new synthesis procedures that could benefit from the synergy between traditional chemical routes and light-matter interaction processes.

\section{Methods}

Chemical reagents. Ethanol absolute, n-hexane (95\%), Silver nitrate (99\%), LCysteine (96\%), sodium diethyldithiocarbamate (DDTC) (ACS reagent grade), $\mathrm{CHCl}_{3}(99.6 \%)$, HS-PEG-COOH $\left(2100 \mathrm{~g} \mathrm{~mol}^{-1}\right)$, and PBS tablets were purchased from Sigma-Aldrich (Germany) and used as received.

Synthesis of $\mathbf{A g}_{2} \mathbf{S}$ dots. $\mathrm{Ag}_{2} \mathrm{~S}$ dots were prepared as follows: $3 \mathrm{mmol}$ of silver nitrate was poured into a round bottom flask containing $10 \mathrm{~mL}$ of octadecylamine at $160^{\circ} \mathrm{C}$ under gentle stirring and $\mathrm{N}_{2}$ atmosphere. After $10 \mathrm{~min}$, the mixture acquired a metallic blue color. At this stage, $1.5 \mathrm{mmol}$ of $\mathrm{L}$-cysteine was added to the mixture as sulfur source. As result, the color of the mixture turned from blue to black indicating the formation of the heterodimer $(30 \mathrm{~min})$. Then, the sample was cooled down and the product of the reaction was dispersed in $40 \mathrm{~mL}$ of $\mathrm{CHCl}_{3}$. This dispersion was centrifuged at $21,000 \mathrm{~g}$ for $30 \mathrm{~min}$ and the precipitate was collected and dispersed in $\mathrm{CHCl}_{3}$ at a concentration of $1 \mathrm{mg} \mathrm{mL}^{-1}$.

Ultrafast laser irradiation. For ultrafast laser irradiation, we used a Ti:Sapphire amplified (Spitfire from Spectra-Physics) pumped by a Ti:Sapphire oscillator (Tsunami from Spectra-Physics) both operating at $808 \mathrm{~nm}$. The amplifier provides pulses with a repetition rate of $1 \mathrm{kHz}$ and tunable pulse widths in the 50-550 fs range by fine adjustement of the compensating gratings. The average power was controlled by using a set of polarizers and a $\lambda / 2$ waveplate (AQWP10M-980 from Thorlabs). A $45 \mathrm{~cm}$ focal length lens was used to focus the irradiation beam into a hermetically closed quartz cuvette containing the dispersion of $\mathrm{Ag}_{2} \mathrm{~S}$ dots in $\mathrm{CHCl}_{3}$. The $\mathrm{Ag}_{2} \mathrm{~S}$ concentration was set to $1 \mathrm{mg} \mathrm{mL}^{-1}$ in all experiments. The focusing lens was mounted on a translation stage that allowed changing the lens-to-cuvette distance and, hence, the laser spot size in the solution. This, in turn, allowed us to change the laser power density while keeping the average laser power constant. Supplementary Fig. 24 shows, as a representative example, the laser spot size and the corresponding irradiation density as a function of the lens-to-cuvette distance for a fixed irradiation laser power of $0.6 \mathrm{~W}$. The NIR-II emission was continuously registered during the irradiation process by a fiber-coupled spectrometer with enhanced sensitivity in the $900-1700 \mathrm{~nm}$ spectral range (Ocean Optics NIRQUEST212). Real-time temperature measurements during irradiation were performed by means of a thermocouple placed into the solution. The distance between the thermocouple and the laser focal spot was set to $2 \mathrm{~mm}$ in order to avoid direct heating of the thermocouple by the $808 \mathrm{~nm}$ laser beam.

Synthesis and characterization of $\mathrm{Ag}-\mathrm{NP}-\mathrm{free} \mathrm{Ag}_{2} \mathrm{~S}$ dots (neat $\mathrm{Ag}_{2} \mathrm{~S}$ dots). The synthesis of the Ag-NP-free $\mathrm{Ag}_{2} \mathrm{~S}$ dots was carried out by thermal decomposition of the precursor silver diethyldithiocarbamate (AgDDTC). The precursor was synthesized as follows: $0.025 \mathrm{~mol}$ of $\mathrm{AgNO}_{3}$ were dissolved in $200 \mathrm{~mL}$ of bidistilled water produced from Milli-Q water. Later $0.025 \mathrm{~mol}$ of DDTC (diethyldithiocarbamate) were dissolved in $300 \mathrm{~mL}$ of bidistilled water and added to the above solution. The resulting yellow powder was filtered and dried at 60 in vacuum using a rotary evaporator. After that, $25 \mathrm{mg}$ of AgDDTC was dispersed in $5 \mathrm{~mL}$ of 1-dodecanethiol and the mixture was stirred under vacuum for $30 \mathrm{~min}$. After that, the solution was heated up to $200^{\circ} \mathrm{C}$ for $1 \mathrm{~h}$. After this time, the solution was cooled down naturally. When the temperature of the mixture reached $25^{\circ} \mathrm{C}, 10 \mathrm{~mL}$ of ethanol was added and the solution was centrifuged at $10,000 \mathrm{rpm}$ for $10 \mathrm{~min}$. The supernatant was discarded and the precipitate collected in $10 \mathrm{~mL}$ of $\mathrm{CHCl}_{3}$.

Ligand exchange procedure. To use the $\mathrm{Ag}_{2} \mathrm{~S}$ superdots for in vivo imaging, we transferred them from $\mathrm{CHCl}_{3}$ to water by a ligand exchange reaction between the octadecylamine and HS-PEG-COOH ( $\mathrm{Mw}$ of $2100 \mathrm{~g} \mathrm{~mol}^{-1}$ ). $2 \mathrm{mg}$ of $\mathrm{Ag}_{2} \mathrm{~S}$ superdots dispersed in $1 \mathrm{~mL}$ of $\mathrm{CHCl}_{3}$ were mixed with $1 \mathrm{mg}$ of $\mathrm{HS}-\mathrm{PEG}-\mathrm{COOH}$ and sonicated for $5 \mathrm{~min}$ to facilitate the ligand exchange. Afterwards, the $\mathrm{CHCl}_{3}$ was gently evaporated until reaching a total volume of $500 \mu \mathrm{L}$. Then, $500 \mu \mathrm{L}$ of absolute ethanol were added and the dispersion was sonicated for $5 \mathrm{~min}$. This process was repeated four additional times in order to ensure the maximum removal of $\mathrm{CHCl}_{3}$. The $\mathrm{Ag}_{2} \mathrm{~S}$ superdots dispersed in $1 \mathrm{~mL}$ of absolute ethanol were then concentrated to a volume of $100 \mu \mathrm{L}$ and transferred dropwise to $900 \mu \mathrm{L}$ of PBS, prepared by dissolving a tablet in $100 \mathrm{~mL}$ of Milli-Q water.

Scanning transmission electron microscopy (STEM). For the STEM studies, a JEOL ARM200 cF was used. This microscope is equipped with a Cs corrector in the condenser lens and an OXFORD INCA detector of $100 \mathrm{~mm}^{2}$ for XEDS analysis. The experiments were carried out at $80 \mathrm{kV}$ as the sample was beam sensitive to higher acceleration voltages. As a first step, sample stability was carefully checked in function of the different parameters of the microscope. To get the highest possible intensity in the XEDS detector without damaging the sample, we determined that the optimum conditions were achieved by using a condenser lens aperture of 50 micrometers and a spot size of 6 . In these conditions, the probe current is well above $120 \mathrm{pA}$. The camera length for the STEM images was set to 6 $\mathrm{cm}$ so that the collection angles range from 90 to $370 \mathrm{mrad}$.

Dynamic light scattering (DLS) and Z-potential determination. The DLS and Z potential measurements were performed using a Zetasizer Nano ZS instrument 
(Malvern Instruments, U.K.). The accumulation time was determined automatically for each sample. The sample concentration was adjusted to obtain 3000 cps. The acquired data were processed using the software provided by Malvern (Zetasizer software v7.03).

Quantum yield measurements. The absolute photoluminescence QY was measured with a calibrated spectrofluorometer (Edinburgh Instruments, FLS920) equipped with an integrating sphere (Jobin-Yvon). A Xe lamp was used as the excitation source, filtered with a longpass filter $(610 \mathrm{~nm})$ and a monochromator (wavelength: $808 \mathrm{~nm}$, bandwidth: $20 \mathrm{~nm}$ ), and a liquid-nitrogen-cooled NIR photomultiplier tube (Hamamatsu, R5509-72) was used for detection. The QY was calculated by dividing the total number of emitted photons in the 900-1700 nm range by the total number of absorbed photons at $808 \mathrm{~nm}$. A representative example of the excitation and emission spectra used for the determination of the QY is shown in Supplementary Fig. 7.

Luminescence decay curves. Luminescence decay curves were obtained by exciting the samples with an OPO oscillator pumped by a frequency doubled $\mathrm{Nd}$ : YAG laser (Lotis), which provides $8 \mathrm{~ns}$ pulses at a repetition rate of $10 \mathrm{~Hz}$. The fluorescence intensity was detected with a Peltier-cooled photomultiplier tube with enhanced sensitivity in the NIR-II (Hamamatsu R5509-73). The contribution of scattered laser radiation was removed with two longpass filters (Thorlabs FEL850) and a high brightness monochromator (Andor Shamrock 320). The time evolution of the fluorescence signal was recorded and averaged by a digital oscilloscope (Le Croy Waverunner 6000).

In vivo imaging. NIR-II in vivo images were obtained in a custom-made NIR-II imaging system. A fiber-coupled diode laser operating at $808 \mathrm{~nm}$ was used as excitation source (LIMO30-F200-DL808). The illumination power density was controlled by adjusting the diode current. The anesthetized mouse was placed on a temperature-controlled plate set to $36^{\circ} \mathrm{C}$. The NIR-II fluorescence images were acquired with a Peltier-cooled InGaAs camera (Xenics Xeva 320) cooled down to $-40{ }^{\circ} \mathrm{C}$. Two longpass filters (Thorlabs FEL850) were used to remove the background signal generated by the scattered laser radiation. In addition, in vivo images were acquired using an additional $1100 \mathrm{~nm}$ longpass filter to ascertain the effect of further spectral filtering on the spatial resolution of the NIR-II images (see Supplementary Figs. 25 and 26)

In vivo experiments were approved by the regional authority for animal experimentation of Comunidad de Madrid and were conducted in agreement with the Universidad Autónoma de Madrid (UAM) Ethics Committee, in compliance with the European Union directives 63/2010UE and Spanish regulation RD 53/ 2013. Experiments were designed in order to use the minimal amount of animals, in accordance with the 3 Rs ethical principle. No randomization or blind studies were performed. For this study, $15 \mathrm{CD} 1$ female mice (8-14 weeks old, weighing $25-39 \mathrm{~g}$ ) bred at the animal facility at UAM were used. Mice were anesthetized prior to the imaging experiments in an induction chamber with a continuous flow of $4 \%$ isoflurane (Forane, AbbVie Spain, S.L.U) in 100\% oxygen until loss of righting reflex was confirmed and breathing rhythm was significantly slowed. Anesthesia was maintained throughout the experiments by means of facemask inhalation of $1.5 \%$ isoflurane and core body temperature was kept at $36 \pm 1^{\circ} \mathrm{C}$, as measured with a rectal probe, using a heating pad.

To study the performance of $\mathrm{Ag}_{2} \mathrm{~S}$ superdots as contrast agents in bioimaging when compared to other NIR-II probes, four mice were shaven and subcutaneously injected with $100 \mu \mathrm{L}$ of a $1.5 \mathrm{mg} \mathrm{mL}^{-1} \mathrm{NP}\left(\mathrm{Ag}_{2} \mathrm{~S}\right.$ superdots, $\mathrm{Ag}_{2} \mathrm{~S}$ dots, $\mathrm{LaF}_{3}: \mathrm{Nd}^{3+}$ or SWNTs) dispersion in PBS.

To study the biodistribution of the $\mathrm{Ag}_{2} \mathrm{~S}$ superdots, two additional mice received a small incision in the neck skin to expose the right jugular vein. A polyethylene tubing catheter was inserted $2 \mathrm{~mm}$ in the caudal direction to infuse $100 \mu \mathrm{L}$ of solution containing $1.5 \mathrm{mg} \mathrm{mL}^{-1}$ PEG-coated $\mathrm{Ag}_{2} \mathrm{~S}$ superdots dispersed in PBS. Three hours after infusion, mice were euthanized by isoflurane overdose and organs (liver, spleen, heart, and lungs) were collected to obtain ex vivo NIR-II images.

One additional mouse was anesthetized in order to check the laser-induced thermal loading with and without fur. In this case, the temperature at the skin surface was measured with an infrared thermographic camera (Fluke iT10).

In vivo biocompatibility studies. Four additional mice were intraperitoneally injected with $300 \mu \mathrm{L}$ of a $500 \mu \mathrm{g} \mathrm{mL}^{-1}$ solution of $\mathrm{Ag}_{2} \mathrm{~S}$ superdots in PBS. Four mice intraperitoneally injected with $300 \mu \mathrm{L}$ of PBS were used as control group. The animals were housed in two separated cages in a room $\left(23 \pm 2{ }^{\circ} \mathrm{C}\right)$ maintained on a $12 / 12 \mathrm{~h} \mathrm{light} /$ dark cycle with free access to food and water. Weight, food intake, and surface temperature, as measured by an infrared thermographic camera (FLIR E-40), were analysed in the awake mice on a daily basis for 2 weeks.

In vivo subchronic toxicological studies. Fifteen mice were intravenously injected with $100 \mu \mathrm{L}$ of a $0.15 \mathrm{mg} \mathrm{mL}^{-1}$ dispersion of $\mathrm{Ag}_{2} \mathrm{~S}$ superdots and euthanized in groups of three at $24 \mathrm{~h}, 7,14,21$, and 28 days post injection. Two additional groups $(n=2)$ were injected with PBS and euthanized after $24 \mathrm{~h}$ and 28 days. Serum ALT-
Alanine transaminase, ALP-Alkaline phosphatase, AST-Aspartate transaminase, creatinine, and total bilirubin were determined.

In vitro cytotoxicity experiments. Cytotoxicity of $\mathrm{Ag}_{2} \mathrm{~S}$ nanodots was evaluated in HeLa (human cervical adenocarcinoma), NIH-3T3 (swiss mouse embryo fibroblasts) and IMR-90 (human lung fibroblasts) using the thiazolyl blue tetrazolium bromide (3-(4,5-dimethyl-thiazol-2yl)-2,5-diphenyltetrazolium bromide, MTT) assay. Briefly, cells in complete medium (DMEM with $10 \%$ FCS, $1 \mathrm{mM}$ pyruvate, $2 \mathrm{mM}$ glutamine, $100 \mathrm{U} \mathrm{mL}^{-1}$ penicillin, $100 \mu \mathrm{g} \mathrm{mL}^{-1}$ streptomycin, and $50 \mu \mathrm{g} \mathrm{mL}^{-1}$ gentamicin) were seeded in 96-well culture plates at a density of $10^{4}$ cells per well After $24 \mathrm{~h}$ of incubation $\left(37^{\circ} \mathrm{C}, 5 \% \mathrm{CO}_{2}\right)$. Cells have been tested for mycoplasma contamination. $\mathrm{Ag}_{2} \mathrm{~S}$ nanodots were added to cells at different concentrations up to $200 \mu \mathrm{g} \mathrm{mL}^{-1}$ and further incubated for $48 \mathrm{~h}$. Then, MTT solution was added for $4 \mathrm{~h}$ to the plate and afterwards, MTT reaction was stopped by adding a solution of dimethylformamide-SDS. Finally, the plate was gently shaken for $2 \mathrm{~h}$ to dissolve formazan crystals prior to measure $570 / 590 \mathrm{~nm}$ absorbance in an Appliskan (Thermo Scientific) plate reader. Corrected absorbance was transformed to percentage of cell viability using the following formula:

$$
\% \text { Cell viability }=\frac{\text { Abs sample }}{\text { Abs control }} \times 100,
$$

where $A b s$ is the corrected absorbance at $570 \mathrm{~nm}$ after subtracting the absorbance at $590 \mathrm{~nm}$.

Experiments were carried out four times and data were represented as a mean \pm standard error.

Reporting summary. Further information on research design is available in the Nature Research Reporting Summary linked to this article.

\section{Data availability}

Source data for all figures are available with the paper. All other data generated and analysed from this study are available from the corresponding authors upon reasonable request.

Received: 11 May 2019; Accepted: 24 April 2020; Published online: 10 June 2020

\section{References}

1. Alivisatos, A. P. Perspectives on the physical chemistry of semiconductor nanocrystals. J. Phys. Chem. 100, 13226-13239 (1996).

2. Bruchez, M., Moronne, M., Gin, P., Weiss, S. \& Alivisatos, A. P. Semiconductor nanocrystals as fluorescent biological labels. Science 281, 2013-2016 (1998).

3. Chan, W. C. \& Nie, S. Quantum dot bioconjugates for ultrasensitive nonisotopic detection. Science 281, 2016-2018 (1998).

4. Naumova, A. V., Modo, M., Moore, A., Murry, C. E. \& Frank, J. A. Clinical imaging in regenerative medicine. Nat. Biotechnol. 32, 804-818 (2014)

5. Dabbousi, B. et al. (CdSe) $\mathrm{ZnS}$ core-shell quantum dots: synthesis and characterization of a size series of highly luminescent nanocrystallites. J. Phys. Chem. B 101, 9463-9475 (1997).

6. Gerion, D. et al. Synthesis and properties of biocompatible water-soluble silica-coated CdSe/ZnS semiconductor quantum dots. J. Phys. Chem. B 105, 8861-8871 (2001).

7. Michalet, X. et al. Quantum dots for live cells, in vivo imaging, and diagnostics. Science 307, 538-544 (2005).

8. Smith, A. M., Mancini, M. C. \& Nie, S. Second window for in vivo imaging. Nat. Nanotechnol. 4, 710-711 (2009).

9. Troy, T. L. \& Thennadil, S. N. Optical properties of human skin in the near infrared wavelength range of 1000 to $2200 \mathrm{~nm}$. J. Biomed. Opt. 6, 167-176 (2001).

10. Hong, G., Antaris, A. L. \& Dai, H. Near-infrared fluorophores for biomedical imaging. Nat. Biomed. Eng. 1, 0010 (2017).

11. Cao, J. et al. Recent progress in NIR-II contrast agent for biological imaging. Front. Bioeng. Biotechnol. 7, 487 (2019).

12. Antaris, A. L. et al. A small-molecule dye for NIR-II imaging. Nat. Mater. 15, 235-242 (2016)

13. Ma, Z. et al. A theranostic agent for cancer therapy and imaging in the second near-infrared window. Nano Res. 12, 273-279 (2019).

14. Tian, R. et al. Multiplexed NIR-II probes for lymph node-invaded cancer detection and imaging-guided surgery. Adv Mater. 32, 1907365 (2020).

15. Li, Y., Zeng, S. \& Hao, J. Non-invasive optical guided tumor metastasis/vessel imaging by using lanthanide nanoprobe with enhanced down-shifting emission beyond $1500 \mathrm{~nm}$. ACS Nano 13, 248-259 (2019).

16. Wang, S. et al. Anti-quenching NIR-II molecular fluorophores for in vivo high-contrast imaging and pH sensing. Nat. Commun. 10, 1058 (2019). 
17. Hong, G. et al. Through-skull fluorescence imaging of the brain in a new nearinfrared window. Nat. Photonics 8, 723 (2014).

18. Wan, H. et al. A bright organic NIR-II nanofluorophore for three-dimensional imaging into biological tissues. Nat. Commun. 9, 1171 (2018).

19. Li, L. et al. A RAtionally Designed Semiconducting Polymer Brush for NIR-II imaging-guided light-triggered remote control of CRISPR/Cas9 genome editing. Adv. Mater. 31, 1901187 (2019).

20. Tian, R. et al. Rational design of a super-contrast NIR-II fluorophore affords high-performance NIR-II molecular imaging guided microsurgery. Chem. Sci. 10, 326-332 (2019).

21. Das, P., Santos, S., Park, G. K., Hoseok, I. \& Choi, H. S. Real-time fluorescence imaging in thoracic surgery. Korean J. Thorac. Cardiovasc. Surg. 52, 205-220 (2019).

22. Bruns, O. T. et al. Next-generation in vivo optical imaging with short-wave infrared quantum dots. Nat. Biomed. Eng. 1, 0056 (2017).

23. Wang, C. et al. Facile aqueous phase synthesis of biocompatible and fluorescent $\mathrm{Ag}_{2} \mathrm{~S}$ nanoclusters for bioimaging: tunable photoluminescence from red to near infrared. Small 8, 3137-3142 (2012).

24. Jiang, P., Zhu, C.-N., Zhang, Z.-L., Tian, Z.-Q. \& Pang, D.-W. Water-soluble $\mathrm{Ag}_{2} \mathrm{~S}$ quantum dots for near-infrared fluorescence imaging in vivo. Biomaterials 33, 5130-5135 (2012).

25. del Rosal, B. et al. In vivo contactless brain nanothermometry. Adv. Funct. Mater. 28, 1806088 (2018).

26. Yang, $\mathrm{T}$. et al. Size-dependent $\mathrm{Ag}_{2} \mathrm{~S}$ nanodots for second near-infrared fluorescence/photoacoustics imaging and simultaneous photothermal therapy. ACS Nano 11, 1848-1857 (2017).

27. Santos, H. D. et al. In vivo early tumor detection and diagnosis by infrared luminescence transient nanothermometry. Adv. Funct. Mater. 28, 1803924 (2018).

28. Ortgies, D. H. et al. Infrared fluorescence imaging of infarcted hearts with $\mathrm{Ag}_{2} \mathrm{~S}$ nanodots. Nano Res. 12, 749-757 (2019).

29. $\mathrm{Li}, \mathrm{C}$. et al. In vivo real-time visualization of tissue blood flow and angiogenesis using $\mathrm{Ag}_{2} \mathrm{~S}$ quantum dots in the NIR-II window. Biomaterials 35 , 393-400 (2014).

30. Li, C. et al. Real-time monitoring surface chemistry-dependent in vivo behaviors of protein nanocages via encapsulating an NIR-II $\mathrm{Ag}_{2} \mathrm{~S}$ quantum dot. ACS Nano 9, 12255-12263 (2015).

31. Hong, G. et al. Multifunctional in vivo vascular imaging using near-infrared II fluorescence. Nat. Med 18, 1841-1846 (2012).

32. Zhang, Y., Liu, Y., Li, C., Chen, X. \& Wang, Q. Controlled synthesis of $\mathrm{Ag}_{2} \mathrm{~S}$ quantum dots and experimental determination of the exciton Bohr radius. $J$. Phys. Chem. C. 118, 4918-4923 (2014).

33. Giansante, C. \& Infante, I. Surface traps in colloidal quantum dots: a combined experimental and theoretical perspective. J. Phys. Chem. Lett. 8, 5209-5215 (2017).

34. Pons, T. et al. On the quenching of semiconductor quantum dot photoluminescence by proximal gold nanoparticles. Nano Lett. 7, 3157-3164 (2007).

35. Tang, A. et al. Controllable synthesis of silver and silver sulfide nanocrystals via selective cleavage of chemical bonds. Nanotechnology $\mathbf{2 4}$ 355602 (2013)

36. Mock, J., Barbic, M., Smith, D., Schultz, D. \& Schultz, S. Shape effects in plasmon resonance of individual colloidal silver nanoparticles. J. Chem. Phys. 116, 6755-6759 (2002).

37. Werner, D., Furube, A., Okamoto, T. \& Hashimoto, S. Femtosecond laserinduced size reduction of aqueous gold nanoparticles: in situ and pump- probe spectroscopy investigations revealing Coulomb explosion. J. Phys. Chem. C. 115, 8503-8512 (2011)

38. Bhushan, B., Kundu, T. \& Singh, B. P. Two-photon absorption spectrum of silver nanoparticles. Opt. Commun. 285, 5420-5424 (2012).

39. Scop, P. M. Band structure of silver chloride and silver bromide. Phys. Rev. 139, A934-A940 (1965).

40. Mahmoud, M. A., Chamanzar, M., Adibi, A. \& El-Sayed, M. A. Effect of the dielectric constant of the surrounding medium and the substrate on the surface plasmon resonance spectrum and sensitivity factors of highly symmetric systems: silver nanocubes. J. Am. Chem. Soc. 134, 6434-6442 (2012).

41. Purnell, J., Snyder, E., Wei, S. \& Castleman, A. Jr Ultrafast laser-induced Coulomb explosion of clusters with high charge states. Chem. Phys. Lett. 229, 333-339 (1994).

42. Naczynski, D. J. et al. Rare-earth-doped biological composites as in vivo shortwave infrared reporters. Nat. Commun. 4, 2199-2199 (2013).

43. Welsher, K., Sherlock, S. P. \& Dai, H. Deep-tissue anatomical imaging of mice using carbon nanotube fluorophores in the second near-infrared window. Proc. Natl Acad. Sci. USA 108, 8943-8948 (2011).
44. del Rosal, B. et al. Overcoming autofluorescence: long lifetime infrared nanoparticles for time-gated in vivo imaging. Adv. Mater. 28, 10188-10193 (2016).

45. Bhavane, R., Starosolski, Z., Stupin, I., Ghaghada, K. B. \& Annapragada, A. NIR-II fluorescence imaging using indocyanine green nanoparticles. Sci. Rep. 8, 14455 (2018).

46. Carr, J. A. et al. Shortwave infrared fluorescence imaging with the clinically approved near-infrared dye indocyanine green. Proc. Natl Acad. Sci. 115, 201718917 (2018).

47. Zhu, S. et al. Repurposing cyanine NIR-I dyes accelerates clinical translation of near infrared II (NIR II) bioimaging. Adv. Mater. 30, 1802546 (2018).

48. Institute ANS. American National Standard for Safe use of Laser. (Laser Institute of America, Orlando, FL, 2007). ANSI Z136.1.

49. Nie, S. Understanding and overcoming major barriers in cancer nanomedicine. Nanomedicine 5, 523-528 (2010).

50. Chenghao, L., Guihuan, C., Bing, Y. \& Hailin, C. Recent advances of low biological toxicity $\mathrm{Ag}_{2} \mathrm{~S}$ QDs for biomedical application. Adv. Eng. Mater. 20, 1700940 (2018).

51. Zhang, Y. et al. Biodistribution, pharmacokinetics and toxicology of $\mathrm{Ag}_{2} \mathrm{~S}$ near-infrared quantum dots in mice. Biomaterials 34, 3639-3646 (2013).

\section{Acknowledgements}

Authors thank Dr A. Benayas (CICECO, U. Aveiro, Portugal), Prof G. Lifante and Prof J. García Sole (UAM) for helpful discussions. This work has been founded by Ministerio de Economía y Competitividad-MINECO (MAT2017-83111R and MAT2016-75362-C3-1-R) and the Comunidad de Madrid (B2017/BMD-3867 RENIM-CM) co-financed by European Structural and Investment Fund. D.M.-G. thanks UCM-Santander for a predoctoral contract (CT17/17-CT18/17). We thank the staff at the ICTS-National Centre for Electron Microscopy at the UCM for the help in the electron microscopy studies and C.M. at the beamline BL22-CLAESS of the Spanish synchrotron ALBA for his help in the XANES experiments. We also thank J.G.I at the Ultrafast Laser Laboratory at UCM for his help and fruitful discussion. Y.S. acknowledges the support from the China Scholarship Council (CSC File No. 201806870023). Additional funding was provided by the European Commission Horizon 2020 project NanoTBTech, the Fundación para la Investigación Biomédica del Hospital Universitario Ramón y Cajal project IMP18_38 (2018/0265). Ajoy K. Kar and Mark D. Mackenzie acknowledge support from the UK Engineering and Physical Sciences Research Council (Project CHAMP, EP/M015130/1). C. Jacinto thanks the financial support of the Brazilian agencies: CNPq (Conselho Nacional de Desenvolvimento Científico e Tecnológico) through the grants: Projeto Universal Nr. 431736/2018-9 and Scholarship in Research Productivity 1C under the Nr. 304967/20181; FINEP (Financiadora de Estudos e Projetos) through the grants INFRAPESQ-11 and INFRAPESQ-12; FAPEAL (Fundação de Amparo à Pesquisa do Estado de Alagoas) grant Nr. 1209/2016. H. D. A. Santos was supported by a graduate studentship from CNPq and by a sandwich doctoral program (PDSE-CAPES) developed at Universidad Autonoma de Madrid, Spain, Project Nr. 88881/2016-01.

\section{Author contributions}

I.Z., M.L., D.M.-G., E.L.C., and J.R.R. fabricated the $\mathrm{Ag}_{2}$ S dots. J.R.R., S.M., O.G.C., M.D.M. A.K.K., and D.J. designed and performed the ultrafast laser irradiation experiments. H.D.A.S., J.R.R., S.M., O.G.C., C.J. and D.J. characterized the optical properties of $\mathrm{Ag}_{2} \mathrm{~S}$ dots and superdots. J.M.-H., C.M.S.J., and D.J. performed the quantum yield measurements. H.D.A.S., Y.S., J.L., E.X., N.F., L.M., B.delR. and D.J. designed and performed the in vivo experiments. I.C.-C., D.L.-A., J.L., and N.F. designed and performed the in vitro and in vivo biocompatibility experiments. J.R.R., B.del.R., and D.J. wrote the paper. H.D.A.S., I.Z.G., Y.S., J.L. and E.X. contributed equally to this work.

\section{Competing interests}

The authors declare no competing interests.

\section{Additional information}

Supplementary information is available for this paper at https://doi.org/10.1038/s41467 020-16333-2.

Correspondence and requests for materials should be addressed to J.R.-R. or D.J.

Peer review information Nature Communications thanks Hak Soo Choi and the other, anonymous, reviewer(s) for their contribution to the peer review of this work. Peer reviewer reports are available.

\section{Reprints and permission information is available at http://www.nature.com/reprints}

Publisher's note Springer Nature remains neutral with regard to jurisdictional claims in published maps and institutional affiliations. 
(c) (i) Open Access This article is licensed under a Creative Commons Attribution 4.0 International License, which permits use, sharing, adaptation, distribution and reproduction in any medium or format, as long as you give appropriate credit to the original author(s) and the source, provide a link to the Creative Commons license, and indicate if changes were made. The images or other third party material in this article are included in the article's Creative Commons license, unless indicated otherwise in a credit line to the material. If material is not included in the article's Creative Commons license and your intended use is not permitted by statutory regulation or exceeds the permitted use, you will need to obtain permission directly from the copyright holder. To view a copy of this license, visit http://creativecommons.org/ licenses/by/4.0/.

(C) The Author(s) 2020 